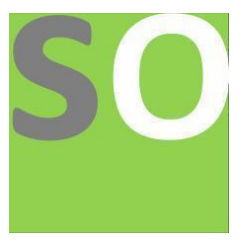

Article title: Binary Data Matrix Theory

Authors: Manouchehr Amiri[1]

Affiliations: Tehran Azad University, Tehran, Iran[1]

Orcid ids: 0000-0002-0884-8853[1]

Contact e-mail: manoamiri@gmail.com

License information: This work has been published open access under Creative Commons Attribution License http://creativecommons.org/licenses/by/4.0/, which permits unrestricted use, distribution, and reproduction in any medium, provided the original work is properly cited. Conditions, terms of use and publishing policy can be found at https://www.scienceopen.com/.

Preprint statement: This article is a preprint and has not been peer-reviewed, under consideration and submitted to ScienceOpen Preprints for open peer review.

DOI: 10.14293/S2199-1006.1.SOR-.PP87C3T.v1

Preprint first posted online: 23 February 2022

Keywords: Quantum Liouville equation, metric compatibility condition, Joint probability, Binary Data Matrix, Ricci flow 


\title{
Binary Data Matrix Theory
}

\author{
Manouchehr Amiri ${ }^{1}$ \\ Islamic Azad University, Tehran, Iran
}

In this paper after introducing a model of binary data matrix (BDM) for physical parameters of an evolving system (of particles), we develop a Hilbert space as an ambient space to derive induced metric tensor on embedded parametric manifold identified by associated joint probabilities of particles observables (parameters). Parameter manifold assumed as space-like hypersurface evolving along time axis, an approach that resembles $3+1$ formalism of ADM and numerical relativity. We show the relation of endowed metric with related density matrix. Identification of system density matrix by this metric tensor, leads to the equivalence of quantum Liouville equation and metric compatibility condition $\nabla_{k} g_{i j}=0$ while covariant derivative of metric tensor has been calculated respect to Wick rotated time or spatial coordinates. After deriving a formula for expected energy per particles, we prove the equality of this expected energy with local scalar curvature of related manifold. We show the compatibility of BDM model with Hamilton-Jacobi formalism and canonical forms. On the basis of the model, I derive the Ricci flow like dynamics as the governing dynamics and subsequently derive the action of BDM model and Einstein field equations. Given examples clarify the compatibility of the results with well-known principles such as equipartition energy principle and Landauer's principle. This model provides a background for geometrization of quantum mechanics compatible with curved manifolds and information geometry. Finally, we conclude a "bit density principle" which predicts the Planck equation, De Broglie wave particle relation, $E=m c^{2}$, Beckenstein bound and Bremermann limit.

Keywords: Quantum Liouville equation; metric compatibility condition; Joint probability; Binary Data Matrix; Ricci flow.

\section{Introduction}

Liouville theorem in statistical physics was first introduced by Joseph Liouville. Theorem states that the density of particles in a system with Hamiltonian regime through time evolution, remains constant in phase space, i.e. $\frac{d \rho}{d t}=0$ [1] .The quantum version of this theorem, namely Liouville -Von Neumann theorem presented in density matrix formalism [2]. Density matrix evolution in Liouville -Von Neumann theorem could be derived directly from Schrödinger equation and acts on the same Hilbert space where the wave function and related operators are defined. This equation is in analogy with the evolution of classical phase space distribution by replacing the density matrix with phase space distribution and commutator with Poisson bracket. One of the major differences between classical and quantum measurement is the limitations induced by Heisenberg uncertainty law and its consequences that constrains the accuracy of joint (simultaneous) measurements of incompatible observables and divides the observables to compatible and incompatible category. Compatible observable refers to those that their operators are commutative and hence could be measured simultaneously while incompatibles are non-commutative and their precise simultaneous measurements are impossible. In spite of this restriction, recent advents reveal some solution for this constraints by imposing some approximations on

\footnotetext{
${ }^{1}$ manoamiri@gmail.com
} 
joint measurements of incompatible observables at the price of introducing some errors with respect to the ideal measurement [3-5]. Then one may consider experiments with acceptable simultaneous measurements of incompatibles with definite concept of joint probability. However joint probability in quantum mechanics remains as an old and challenging area of research. One of the main approaches for quantum correction to classical statistical mechanics and consequently joint probability in quantum mechanics are brought by Wigner distribution (function) to formulate the quantum physics in a phase space through introduction of Quasi probabilities. The Quasi prefix is considered because of emerging some negative probabilities in the context of Wigner distribution. It has been proved that these negative probabilities often refer to small parts of phase space and could be ignored in most problems [6]. Actually whenever the Wigner function takes just the non-negative values it represents a true joint probability distribution of position and momentum [6]. At the time being joint measurements of incompatible observable with some error becomes feasible [5]. Therefore, implementing joint measurements to record the magnitude of observables with a possible range of errors is achievable. This means that one may define joint probabilities in quantum approach especially in density matrix formalism[5]. We will present in sec (3) a density matrix which fitted for the present model with entries proportional to joint probabilities of observables. In sec $(2,3,4)$ we set a model of a binary data matrix $\boldsymbol{D}$ which contains evolving data of parameters of all particles in a system with 0 and 1 entries. The rows of this matrix are base vectors in the Hilbert space $\mathcal{H}_{2}$ and their inner products constitute a metric tensor for dual space of the parametric space. We will show in these sections the equivalence of density matrix with a symmetric matrix $\boldsymbol{d d}^{T}$ which derived from $\boldsymbol{D}$.

Metric compatibility condition exhibited as a pure mathematical inference in differential geometry and tensor analysis [7].This theorem states that for any chosen local coordinates the covariant derivative of metric tensor $g_{i j}$ vanishes i.e. $\nabla_{k} g_{i j}=\nabla_{k} g^{i j}=0$ [8]. When we apply the covariant time derivative of metric tensor (after wick rotation), the metric compatibility condition and quantum Liouville equation as two apparently far concepts appear as two sides of a common reality when the deep connection of metric tensor and joint probability has been shown to be based on an abstract background of evolution process of a system of large number of particles. The consequences of this equivalence result in a definition for energy per particle with ensuing equations of action integral and Einstein field equations. The transition from discrete particles continuous derivative and connections are the same method in MaxwellBoltzmann kinetic theory of gases. The binary matrix model geometrizes the statistical concepts in physical parameter space based on binary data of system. In some approach to general relativity like numerical relativity and ADM formalism [9], the concept of foliation of space-time manifolds into space like hypersurfaces has been introduced and used to solve some related problems. These hyper-surfaces embedded in space-time manifold with time-like unit normal vectors. We generalize this method to $n+1$ dimensional parametric manifolds with hyper-surfaces of $n$ space-like dimensions of physical parameters. Accordingly, in this approaches the hyper-surfaces and their induced metrics could be evolved through time under quantum Liouville equation. In sec (3) we would have shown the equivalence of density matrix and metric tensor of parametric space and its dual space with joint probabilities of particle parameters which appears as a symmetric matrix $\boldsymbol{d} \boldsymbol{d}^{T}$ derived from binary data matrix $\boldsymbol{D}$. In section (6) we prove the Ricci flow dynamic as a direct consequence of the context of binary matrix model and then apply it in action integral to derive Einstein field equations. Ricci flow is a well-known geometric flow was first introduced by Hamilton and used for solution of the Poincare conjecture. As an evolution equation of metric tensor, Hamilton (1982) showed the existence of unique solution of Ricci flow equation on a closed manifold over a sufficiently short time. Mainstay of general relativity has been based on the relation of space-time manifold structure and stress energy tensor in the presence of gravitational field by presumption of equivalence principle [10]. Einstein field equation represents this equivalence by equating a pure geometrical term (left side) well known as Einstein tensor with a pure physical term (i.e. stress 
energy tensor) [10]. This great assumption leads to geometrization of all gravitational and nongravitational field theories through introduction of Einstein-Hilbert action integral in such a way that metric tensor of space-time appears in all actions of field theories. In recent years some attempts devoted to introduce the gravity as an emerging force i.e. entropic force [11]. By these scenarios the distribution of mass-energy dictates the gravitational potential [11, 12]. These theories support the relation of geometry and probability. Intuitively an immediate result is a probable deep connection between the geometry (of space-time) and physical probability concept. As an interesting example we have shown in sec (8) the more basic interconnection between joint probability density (as an induced metric tensor) and the Einstein tensor under Ricci flow dynamic. Geometrization of probability distribution and information has been achieved by some authors. Historically, some attempts toward the geometrization of statistical inferences and probability distributions, have been made Amari and Fisher to develop the metric tensor concept of manifolds constructed by points correspond to probability distributions in order to geometrize the information theory. Fisher information and covariance based metric in phase space and information geometry are among the original works in this field and their applications in thermodynamics $[13,14]$. However, these approaches limited to phase space with definitions of metric tensor as the expectation values of probability distribution moments and likelihoods. Moreover, there has not revealed a clear connection to physical applications. Therefor local approaches have not been yet developed properly in order to be used in Riemannian curved spaces and general relativity. Some authors also indicated the relations of thermodynamic rules with Einstein field equations $[15,16]$. These theories describe the gravitational forces with entropic force assuming entropy as a function of matter distribution [12]. Although the pure geometrical part of Einstein field equations could be served in arbitrary dimensional space, however its physical side should be realized in four-dimensional space-time continuum, accordingly it seems to be a special case of a more general form of basic laws. In this article we generalize the physical concept of geometrical part of Einstein field equation in $n+1$ dimensional manifolds defined through exploring a deep connection between the concepts of metric and joint probability density. In subsequent sections I describe the wide range consequences of the model which incorporate the quantum mechanics and general relativity by deriving the universe inflation, Schrodinger equation, equipartition energy principle, Landauer's principle and classical thermodynamic laws. As an important result, I conclude the bit density principle which unites the De Broglie wave- particle equation, Planck photon energy, and mass- energy relation $E=m c^{2}$. Beckenstein bound and Bremermann limit are straightforward results of this principle. Equivalence of Euclidean action and entropy of black holes are among other consequences of binary matrix model. Binary matrix model initiates with a quantum approach (quantum Liouville equation) and after translating the physical parameter to bit information results in the basic equations of general relativity (Einstein field equation) Universe inflation to reconcile quantum mechanics and general relativity. The approach can be depicted as:

$$
\text { quantum liouville equation }+B D M+\text { information bit } \rightarrow \text { General relativity }
$$

\section{Binary Data Matrices and Hilbert space}

Definition: Parametric space $\mathcal{M}$ specified by $x^{v}$ coordinates with $\boldsymbol{v}$ which varies from 1 to the dimension of parametric space $\mu$ :

$$
1 \leq v \leq \boldsymbol{\mu}
$$

Let construct a binary data matrix on the basis of sequential measurements take place in a time interval $\Delta \mathbb{T}$ on $N$ particles in a system with conserved total number. One may label each particle by a number so that the first measurement implemented on first particle and second measurement on second particle 
and so on. $\Delta \mathbb{T}$ represents the least time required to achieve measurements of all particles and is assumed to be a small time interval. We label these set of measurements by $\{\alpha \mid 1 \leq \alpha \leq N\}$ with time ordering. If our measurements include $\mu$ independent parameters ( $\boldsymbol{\mu}=$ degrees of freedom) are being denoted by $x^{\nu}$ ( $v$ denotes the $v$-th degree of freedom) then we can divide the possible range of these parameters to a large number of intervals $\Delta x^{v}$ in order to obtain such small intervals that satisfy the order of predicted error of measurement setting and the accuracy of measurements. If the number of these intervals for each parameter $x^{v}$ denoted by $m_{v}$, the total number of intervals reads as:

$$
m=\sum_{v} m_{v}
$$

Accordingly any measurement outcome of a particle to determine the value of specific parameter $x^{v}$ falls just in one interval labeled by ' $i$ ' denoting the $i$ th interval meanwhile stands for a specific value of $x^{\nu}$. Let show this interval by $\Delta x^{v}(i)$ and attribute the binary value $\mathbf{1}$ for this interval while the other intervals take the value $\mathbf{0}$. Consequently, the result of $x^{v}$ measurement for a particle will be represented by some column binary matrix with non-zero (1) element only at row specified by $x^{v}(i)$. Iteration of measurement on other parameters turn out other column binary matrices. The outcome of all parameters could be represented by $\mu$ column binary matrix with $\mu$ non-zero entries. Conjunction of these column binary matrices as a single column binary matrix result in a matrix $\xi_{m \times 1}^{\alpha}$. Each of these $\xi_{m \times 1}^{\alpha}$ gives the parameter values of the $\alpha$ th particle. Union of $\xi_{m \times 1}^{\alpha}$ constructs a data matrix $\boldsymbol{D}_{\boldsymbol{m} \times \boldsymbol{N}}$. Rows of these binary data matrices i.e. at each interval $\Delta x^{v}(i)$ can be denoted by a vector $e^{* v}(i)$ :

$$
e^{* v}(i)=(0,1,0,0,1,1,0,0,1, \ldots)
$$

Let call these base vectors as data basis vectors. Each vector $e^{* v}(i)$ could be regarded as a base vector spanned in an abstract $N$ dimensional space with binary components. We will define this $N$ dimensional space as particle-oriented coordinates. Obviously, $\boldsymbol{D}_{\boldsymbol{m} \times \boldsymbol{N}}$ could be partitioned to $D_{m_{v} \times N}$ matrices for each parameter $x^{v}$. Thus, matrix product $\boldsymbol{D} \boldsymbol{D}^{T}$ contains block matrices for each parameter as diagonal entries and block matrices produced by different parameters as non-diagonal entries.

$$
\boldsymbol{D D}^{\boldsymbol{T}}=\left[\begin{array}{cccc}
D_{m_{1} \times m_{1}} & D_{m_{1} \times m_{2}} & \cdots & \\
D_{m_{2} \times m_{1}} & \ddots & & \vdots \\
\vdots & \cdots & & D_{m_{\mu} \times m_{\mu}}
\end{array}\right]
$$

As we will prove in Lemma 2, the entries of this matrix carry the set of joint probabilities of parameters. For space coordinate of particles, the involved block matrices yield the spatial distribution of particles.

Postulate At the limit $\Delta x^{v}(i) \rightarrow d x^{v}(i)$, the vectors $e^{* v}(i)$ approaches the basis of cotangent bundle (space) as 1-form i.e. $\quad e^{* v}(i) \equiv d x^{v}(i) \equiv \omega^{v}(i)$

Definition: Here any particle specifies an independent coordinate with two possible values $\mathbf{0}$ and $\mathbf{1}$. These coordinates are orthogonal, because at the initial setting the parameter values of each particle (such as position and momentum etc.) considered to be independent of all other particles. We call these set of coordinate as particle-oriented coordinate that as a coordinate chart is homeomorphic to a subset of Euclidean flat space $\mathbb{R}^{N}$ which span a manifold $\boldsymbol{M}$. Moreover we define a parametric space $\mathcal{M}$ of considered system including all coordinates $x^{v}$ and their dual basis $e^{* v}$ where the latter span a dual tangential (cotangent) vector space $T_{P}^{*} \mathcal{M}$ at a point $\boldsymbol{p}$ in parametric space $\mathcal{M}$ i.e.

$$
\operatorname{Span}\left\{e^{* v}\right\}=T_{P}^{*} \mathcal{M} \subset \boldsymbol{M}
$$


Lemma 1. It is straight forward to deduce the orthogonality of $e^{* v}(i)$ in each parametric range of $x^{v}$ by scalar products:

$$
\left\langle e^{* v}(i), e^{* \nu}(j)\right\rangle=0 \quad i \neq j
$$

Proof: components of $e^{* v}(i)$ defined in an orthogonal particle-oriented coordinates. Let $n$-th component be denoted by:

$$
\left[e^{* v}(i)\right]_{n}
$$

Then the scaler product in an orthogonal coordinate for a fixed $v$ reads as:

$$
\left\langle e^{* v}(i), e^{* v}(j)\right\rangle=\sum_{n}\left[e^{* v}(i)\right]_{n}\left[e^{* v}(j)\right]_{n}^{T}
$$

If a specific component $\left[e^{* v}(i)\right]_{p}$ takes the value $\mathbf{1}$, this means that the value of parameter $x^{v}$ for $p$-th particle falls in $i$-th interval and other intervals as $\left[e^{* v}(j)\right]_{p}$ could not take the same value, and vice versa, therefore we have:

$$
\left[e^{* v}(j)\right]_{p}=0 \quad i \neq j
$$

Consequently in equation (7) $\left[e^{* v}(i)\right]_{p}$ and $\left[e^{* v}(j)\right]_{p}$ could not take the value 1 simultaneously and this sum as the inner (scaler) product vanishes.

In order to derive a matrix containing the relative and simultaneous abundance of positive interval population (i.e. total number of particles of different parameters) we need to extract all scalar products $e^{* \mu}(i) \cdot\left[e^{* v}(j)\right]^{T}=\left\langle e^{* \mu}(i), e^{* v}(j)\right\rangle$ obtained by means of the matrix product $\boldsymbol{D} \boldsymbol{D}^{\boldsymbol{T}}$.

Lemma 2. Diagonal entries of the matrix $\boldsymbol{D} D^{T}$ are equivalent to the separate probability of each interval and non-diagonal entries return the joint probabilities of different parameter intervals after necessary normalization.

Proof: Elements of $\boldsymbol{D} \boldsymbol{D}^{\boldsymbol{T}}$ could be represented as (the index $\mu$ should not be confused with degree of freedom $\boldsymbol{\mu}$ )

$$
\left\langle e^{* v}(i), e^{* \mu}(j)\right\rangle=\sum_{n}\left[e^{* v}(i)\right]_{n}\left[e^{* \mu}(j)\right]_{n}^{T}
$$

Obviously, this sum enumerates the total number of particles that have common parameter value of $i$-th interval of $x^{\nu}$ and $\boldsymbol{j}$ th interval of $x^{\mu}$. Hence the joint probability of $e^{* \nu}(i)$ and $e^{* \mu}(j)$ events reads as:

$$
f^{\mu \nu}=\frac{1}{N}\left\langle e^{* v}(i), e^{* \mu}(j)\right\rangle
$$

Moreover, for each point on $\mathcal{M}$ the we can retrieve $f^{\mu \nu}$ via matrix multiplication $\boldsymbol{d} \boldsymbol{d}^{T}$ where $\boldsymbol{d}$ is the matrix obtained by collection of row vectors $e^{* \nu}$ of various parameters all defined on a point on $\mathcal{M}$. Thus $\left\|f^{\mu \nu}\right\|=\boldsymbol{d}^{T}$ is a symmetric square matrix of order $\boldsymbol{\mu}$ (the number of parameters), defined on a point on $\mathcal{M}$ :

$$
\boldsymbol{d}=\left[\begin{array}{c}
{\left[e^{* 1}\right]} \\
{\left[e^{* 2}\right]} \\
\cdots \\
{\left[e^{* \mu}\right]}
\end{array}\right] \quad, \quad\left\|f^{\mu v}\right\|=\boldsymbol{d}^{T}
$$

Lemma 3. Paired joint probabilities $f^{\mu v}$ indicate the local metric tensor of $\mathcal{M}$. For each point on $\mathcal{M}$ the $f^{\mu \nu}$ represents a matrix of order $\boldsymbol{\mu} \times \boldsymbol{\mu}$ and is equivalent to metric tensor of parametric space $\mathcal{M}$. 
The general definition of metric tensor for a manifold with local base vectors $e^{* v}(i)$ is compatible with equation (10):

Therefore

$$
\begin{aligned}
& g^{\mu \nu}=\left\langle e^{* v}(i), e^{* \mu}(j)\right\rangle \\
& \frac{1}{N} g^{\mu \nu}=f^{\mu \nu} \\
& \left\|g^{\mu \nu}\right\|_{\boldsymbol{\mu} \times \boldsymbol{\mu}}=\boldsymbol{d} \boldsymbol{d}^{T}
\end{aligned}
$$

The total information bits collected during measurement on such a system with $\mu$ as the degree of freedom will be read as: $\quad \mathbb{N}=\boldsymbol{\mu} N$

In this model we define a Hilbert space $\mathcal{H}$ with all basis of the form $\left\langle j_{1}, j_{2}, \ldots j_{n}\right|$ with $j_{m} \in\{\mathbf{0}, \mathbf{1}\}$. In quantum computation however, these basis well known as (quantum) computational basis vectors (states) of the Hilbert space $\mathcal{H}_{2^{n}} \quad[17,18] .2^{n}$ refers to the total number of elements of this Hilbert space. $\mathcal{H}_{2^{n}}$ contains all $e^{* v}(i)$ and related $\mathcal{N}_{v}$ spaces. The $\mathcal{N}_{v}$ spaces are sub-spaces of $\mathcal{H}_{2^{n}}$ and could be described as Hilbert spaces $\mathcal{H}_{v}$ for each parameter $x^{\nu}$. This approach, is in close relation to qubit basis definition in quantum computation theory[17]. Indeed, for construction of tangent spaces compatible with our model we need to choose a sub-space of base vectors of $\mathcal{H}_{v}$ in such a way that inner product of any pair of them vanishes:

$$
\left\langle e^{* v}(i), e^{* v}(j)\right\rangle=0 \quad i \neq j
$$

Obviously, these sub-spaces may be regarded as $\mathcal{H}_{v}$. Sub-spaces $\mathcal{H}_{v}$ are spanned by $m_{v}$ base vectors $e^{* \nu}(i)$. Then the whole space could be represented as the sum (not direct sum) of sub-spaces $\mathcal{H}_{v}$ :

$$
T_{P}^{*} \mathcal{M}=\mathcal{H}_{1}+\mathcal{H}_{2} \ldots+\mathcal{H}_{\mu} \subset \mathcal{H}
$$

The collected information of system of particles over time interval $\Delta \mathbb{T}$, leads to a binary data matrix $\boldsymbol{D}_{m \times N}$.

Each sub-space $\mathcal{H}_{v}$ considered as a tangent sub-manifold $\mathcal{N}_{v}$ at a point $p$. The union of these tangent spaces results in the total space of a tangent bundle $T_{P}^{*} \mathcal{M}$. The state of the system could be represented by such matrix and the evolution of this quantum system obeys the equation of quantum Liouville theorem as well as Hamiltonian operator. The inner product property of this Hilbert space leads to definition of metric tensor and related curvatures induced on manifold $\mathcal{M}$.

Definition: We have shown Hilbert space $\mathcal{H}$ spanned by $e^{* v}(i)$ as base vectors of related vector space. The "bra" notation determines these bases in the sense of quantum mechanics. If one shows the "bra" with $\left\langle e^{* v}(i)\right|$ then the related dual base vector will be denoted by "ket" i.e. $\left|e_{\widehat{\mu}}^{*}(j)\right\rangle$ and lives in dual vector space $\mathcal{H}^{*}$. In matrix form, $\left\langle e^{* v}(i)\right|$ presented by a row matrix as depicted in equation (3) and $\left|e^{* v}(i)\right\rangle$ by a column matrix that is transpose of $\left\langle e^{* v}(i)\right|$. For compatibility with tensor representation we use reasonably the lower index for "ket" vector and therefore we have $\left|e_{\widehat{\mu}}^{*}(j)\right\rangle$ instead of. $\left|e^{* \widehat{\mu}}(j)\right\rangle$ and the scaler of the "bra" and "ket" in this notation reads as:

$$
\left\langle e^{* v}(i), e_{\mu}^{*}(j)\right\rangle
$$

We sued $\hat{\mu}$ instead of $\mu$ to emphasize that this index refers to the double dual of parametric space while we know the isomorphism of double dual with original vector space [29]. The joint probability as proved in lemma 3 is a tensor because is proportional to metric tensor. In the notation of (15) this joint probability should be shown by a mixed tensor defined in dual and double dual vector space: 


$$
f_{\widehat{\mu}}^{v}=\frac{1}{N}\left\langle e^{* v}(i), e_{\widehat{\mu}}^{*}(j)\right\rangle
$$

The value of scaler product $\left\langle e^{* v}(i), e_{\widehat{\mu}}^{*}(j)\right\rangle$ equals $\left\langle e^{* v}(i), e^{* \mu}(j)\right\rangle$. The upper index of $f_{\widehat{\mu}}^{v}$ related to dual space while the lower index to double dual space. Because of isomorphism between original and double dual space [29] this tensor could be considered as a mixed rank 2 tensor in parametric (original) vector space. Thus, for compatibility with bra and ket notation we apply this tensor as metric tensor evolving by time.

$$
\frac{1}{N} g_{\widehat{\mu}}^{v}=f_{\widehat{\mu}}^{v}=\frac{1}{N}\left\langle e^{* v}(i), e_{\widehat{\mu}}^{*}(j)\right\rangle
$$

\section{Equivalence of metric compatibility condition and quantum Liouville equation}

Density matrix formalism is the quantum version of phase space probability measure of classical statistical mechanics. Accordingly, it deals with ensembles of mixed and pure states. The general definition of density matrix could be read as:

$$
\rho=\sum_{i j}|i\rangle \rho_{i j}\langle j|
$$

$|i\rangle$ denotes the basis vector labelled by " $i$ " and $|i\rangle\langle j|$ denotes the projection matrix with non-zero element at row " $i$ " and column " $j$ ". The corresponding element presented by $\rho_{i j}$. Diagonal entries $\rho_{i i}$ of density matrix represents the population (probability) of a specific basis (state) therefore the trace of density matrix is unit. Off diagonal entries would provide information about the degree of coherence (or polarization) between two states, in other words it represents the correlation of basis states. Although off-diagonal elements have no simple physical interpretation it always gives information on quantum correlation between particles and fields $[19,20]$. we consider these off-diagonal elements as the usual correlations between parameters (random variables) $x_{i}$ and $x_{j}$ which could be encoded by their joint probabilities [20], whereby we assume in our definition the equivalent notion of off-diagonal entries of density matrix $\left(\rho_{\mu \nu}\right)$ and joint probability density function:

$$
\rho_{\mu \nu}=f_{\widehat{\mu}}^{v}=\frac{1}{N} g_{\widehat{\mu}}^{v}
$$

Because the factor $\frac{1}{N}$ is a scaler constant of system, it could be absorbed by $g_{\widehat{\mu}}^{v}$ and from now on we use the term $g_{\widehat{\mu}}^{v}$ instead of $\frac{1}{N} g_{\widehat{\mu}}^{v}$ without any change in dynamics and topology of $\mathcal{M}$.

$$
\rho_{\mu \nu}=f_{\widehat{\mu}}^{v}=g_{\widehat{\mu}}^{v}
$$

In the sense of quantum computation $\langle j|$ vectors are computational basis vector in the form $\left\langle j_{1}, j_{2}, \ldots j_{n}\right|$ with $j_{m} \in\{\mathbf{0}, \mathbf{1}\}$. In present model these vectors substituted by data basis vector $e^{* v}(i)$ which corresponds the $\boldsymbol{i}$ th row of $\boldsymbol{D}_{m_{v} \times N}$ matrix. With the identification of bra $\langle v|$ by $e^{* v}(i)$ and ket $|\mu\rangle$ by $e_{\hat{\mu}}^{*}(j)$ the density matrix entries $\rho_{\mu \nu}$ respect to (19) could be represented by:

$$
\rho_{\mu \nu}(i, j)=\frac{1}{\mathrm{~N}}\left\langle e^{* v}(i), e_{\hat{\mu}}^{*}(j)\right\rangle=f_{\widehat{\mu}}^{v}(i, j)
$$

$i, j$ determine the corresponding intervals (values) of $x^{v}$ and $x^{\mu}$ respectively. The off-diagonal entries give the classical joint probabilities $f_{\widehat{\mu}}^{v}$. 
Recalling the equation (16) also reveals the equivalence of $\rho_{\mu \nu}$ and $g_{\mu \nu}$ and their symmetric and positive definite properties. One may compare these correspondence with similarities of covariance matrix and metric of thermodynamic state manifold [19]. One may use $f_{\hat{\mu}}^{v}$ as a mixed tensor defined by inner product of a base $e^{* v}(i)$ with a dual base $e_{\widehat{\mu}}^{*}(j)$, by the same components of $g_{\widehat{\mu}}^{v}$ as a metric tensor and consequently with vanishing covariant derivative due to metric compatibility. Respect to binary data matrix mentioned in previous section we can imply a new relation between metric compatibility in differential geometry and Liouville equation in quantum density matrix notion. It should be reminded that the trace of defined $\rho_{i j}$ equals the constant $\mu$ (the degree of freedom). Evidently, this fact does not interfere the validity of what will be followed.

Definition: Let $(\mathcal{M}, g)$ stands for a $\boldsymbol{\mu}$ dimensional space-like Riemannian manifold described in sections (1),(2) with $g_{\mu \nu}$ as metric and $f_{\mu \nu}$ as joint probabilities described in section (2). Evolution of particles system evolves this manifold through time axis. The overall manifold $M$ comprises space-like manifolds $\mathcal{M}$ and time coordinate generally constructs a Lorentzian manifold where a Wick rotation (i.e.t $=i c \tau=$ $i \tau$ ) convert it to a Riemannian manifold of dimension $v+1$. Therefore $M$ foliated by hypersurfaces $\mathcal{M}$ through time axis. This approach is close to ADM formalism and numerical relativity [9]. From now on we use alphabetic indices instead of Greek letters. Metric compatibility known as vanishing of covariant derivative of metric tensor i.e. $\nabla_{k} g_{i j}=g_{i j ; k}=0$. Here we use the covariant derivative respect to Wick rotated time axis: $g_{i j ; 0}=0$ because the evolution of these systems occur along the time axis and this reveals the rational for exclusive role of time covariant derivative of metric tensor in comparison with the spatial derivatives. This condition is also valid for metric $g_{\hat{\mu}}^{v}$.

Theorem: For a system of particles and associated manifold $\mathcal{M}$ endowed by the metrics $g_{i j}$ defined in section (2) vanishing covariant derivative of metric tensor (respect to Wick rotated time) is equivalent to quantum Liouville equation.

Proof: Density matrix evolution in quantum setting and its Liouville-von Neumann equation for time evolution with $H_{m j}$ as matrix form of Hamiltonian operator could be read as [2, 21]:

$$
\frac{\partial \rho_{m n}}{\partial t}=-\frac{i}{\hbar} \sum_{j}\left(H_{m j} \rho_{j n}-H_{j n} \rho_{m j}\right)
$$

In the Planck units $\hbar=c=1$ by taking into account the Euclidean coordinate after a Wick rotation i.e. $t=i c \tau=i \tau$ and substituting it in above equation we have:

$$
\frac{\partial \rho_{m n}}{\partial \tau}=\sum_{j}\left(H_{m j} \rho_{j n}-H_{j n} \rho_{m j}\right)
$$

Regarding metric compatibility in differential geometry [4] i.e. $\nabla_{k} g_{i j}=\nabla_{k} g^{i j}=0$ and equation (19) i.e. $\frac{1}{N}$ $f_{n}^{m}=g_{\hat{n}}^{m}=\rho_{m n}$. The joint probability $f_{n}^{m}$ is equivalent to metric tensor $g_{\hat{n}}^{m}$. Taking into consideration the temporal component (covariant derivative of metric tensor respect to Wick rotated time $\tau$ ) of tensor compatibility, by definition of covariant derivative we obtain:

$$
\nabla_{0} g_{\widehat{m}}^{n}=\nabla_{0} f_{m}^{n}=0 \Rightarrow \frac{\partial f_{m}^{n}}{\partial \tau}=\Gamma_{0 m}^{j} f_{j}^{n}-\Gamma_{0 j}^{n} f_{m}^{j}
$$

Then we get (by Einstein summation convention on $j$ index and symmetry of matrix $f_{n}^{m}=f_{m}^{n}$ ): 


$$
\frac{\partial f_{m}^{n}}{\partial \tau}=\Gamma_{0 m}^{j} f_{j}^{n}-\Gamma_{0 j}^{n} f_{m}^{j}
$$

Where $\Gamma_{0 m}^{j}$ terms denote the Christoffel symbols. Comparing equations (22) and (24) reveals a new relation between Christoffel symbol and Hamiltonian matrix of the considered state:

$$
\sum_{l} H_{m l} \rho_{l n}=\sum_{l} H_{m l} f_{l}^{n} \sim \Gamma_{0 m}^{l} f_{l}^{n}
$$

Accordingly we achieve a correspondence: $\quad H_{m j} \sim \Gamma_{0 m}^{j}$

For general strict equation instead (26), one needs an additional constant term to $\Gamma_{0 m}^{j}$ which does not depend on metric tensor, namely:

$$
H_{m j}=\Gamma_{0 m}^{j}+C_{m}^{j}
$$

Then the equation (22) and (24) remain compatible. In next sections taking $C_{m}^{j}=0$, leads Hamiltonian operator to be reduced to $H_{m j}=\Gamma_{0 m}^{j}$. The term $C_{m}^{j}$ stands for a constant trace mixed tensor which independent of indices remains with constant trace i.e.

\section{Derivation of Mean energy}

$$
\operatorname{Tr}\left(C_{m}^{j}\right)=K
$$

Considering the relation of energy expectation value $\langle E\rangle$ of a system with Hamiltonian $\widehat{H}$ and density matrix $\rho_{m j}$ :

$$
\langle E\rangle=\operatorname{Tr}(\rho \widehat{H})=\sum_{m j} H_{m j} \rho_{m j}
$$

With substitution of $H_{m j}$ and $\rho_{m j}$ from (19) and (27) and identity $\Gamma_{0 m}^{j}=g^{j k} g_{k m, 0}$ and using Einstein summation convention we have:

$$
\operatorname{Tr}(\rho \widehat{H})=g_{\hat{\jmath}}^{m}\left(\Gamma_{0 m}^{j}+C_{m}^{j}\right)=\left(g_{\hat{\jmath}}^{m} g^{j k} g_{k m, 0}+g_{\hat{\jmath}}^{m} C_{m}^{j}\right)=\left(g^{m k} g_{k m, 0}+C_{m}^{m}\right)
$$

Using the formula for trace of Christoffel symbol ( $\Gamma_{0 m}^{m}=g^{m k} g_{k m, 0}=\frac{1}{2} \frac{\partial}{\partial \tau} \log g$ ) [10] we get a relation between energy expectation value as trace of $\rho \widehat{H}$ and the trace of $\Gamma_{0 m}^{j}$ as follows:

$$
\langle E\rangle=\operatorname{Tr}(\rho \widehat{H})=\left(\Gamma_{0 m}^{m}+C_{m}^{m}\right)=\frac{1}{2} \frac{\partial}{\partial \tau} \log g+K
$$

Where $\langle E\rangle$ denotes the energy per particle (constituent)[2] at a specific point $P$ where both $\rho_{\mu \nu}$ and $g_{\widehat{\mu}}^{v}$ in equation (19) are defined, $g$ stands for determinant of metric tensor $g_{i j}$. The trace of $C_{m}^{j}$ substituted by $K$ and appears as a constant. Without loss of generality, we could assume $K=0$ then:

$$
\langle E\rangle=\frac{1}{2} \frac{\partial}{\partial \tau} \log g
$$

$K$ as the constant part of particle energy, could be considered the rest mass energy of particle i.e. $K=$ $m_{0} c^{2}$, however it can be omitted in non-relativistic approximations. As described above, $\langle E\rangle$ stands for mean energy per constituent (particle) at an exact interval of parameters (i.e. volume element $d^{n} \boldsymbol{\omega}$ of the related manifold). Since at equilibrium state, each particle contains $\mu$ bit of information, therefore in 
our model $\langle E\rangle$ is equivalent to energy of $\mu$ bit. We will show the consequences of this result in sec (7). Accordingly, the whole expected energy of $N$ particle system at thermal equilibrium, $\mathbb{E}$ can be read as:

$$
\mathbb{E}=N\langle E\rangle=\frac{N}{2} \frac{\partial}{\partial \tau} \log g
$$

The main result of this equation, regarding the energy conservation of system, is a continuous evolution and matric change. Metric of considered system and its determinant $g$ should change by a rate determined by the total energy content of system. If we denote $\boldsymbol{g}_{i j}$ as the corresponding matrices of spatial coordinates in $\boldsymbol{d d}^{T}$ this metric is also involving in time evolution. In a system with equilibrium state respect to parameters other than space parameters, we expect the change rate of determinant $g$ is determined by the time evolution of $\boldsymbol{g}_{i j}$ :

$$
\mathbb{E} \sim \frac{\partial}{\partial \tau} \log \left(\operatorname{detg}_{i j}\right)
$$

The default positive sign of $\mathbb{E}$ yields: $\quad \frac{\partial}{\partial \tau} \log \left(\operatorname{det}_{i j}\right)>0$

This reveals that in any unbounded system there is a tendency toward the expansion of spatial coordinate. We realize this result in section 7.5. Of course, $N\langle E\rangle$ stands for the mean energy of total system consisting of particles or a hierarchy of information bits or the energy density. We will present $\mathcal{M}$ in next sections as a non-compact manifold specified for a class of ensembles with certain energy and particle number.

\section{Canonical formalism Of BDM model}

Taking into account that metric compatibility condition includes all coordinate variables we generalize the previous section discussion by extending the Liouville equation for other physical coordinates. First, we rewrite the general metric compatibility condition:

$$
\nabla_{k} g_{i j}=\nabla_{k} g^{i j}=0
$$

In this section we show that the term introduced as $\varphi=-\frac{1}{2} \log g$ appeared in energy equation of BDM model:

$$
E=\frac{1}{2} \frac{\partial}{\partial t} \log g
$$

Is equivalent to Hamilton principal function in classical mechanics. The equation for energy has been derived from quantum Liouville equation:

$$
\frac{\partial \rho}{\partial t}=-\frac{i}{\hbar}[H, \rho]
$$

Replacing time and Hamiltonian by spatial coordinates and linear momentum respectively gives:

$$
\frac{\partial \rho}{\partial x}=\frac{i}{\hbar}\left[p_{x}, \rho\right]
$$

Evidently this relation also holds for any other degree of freedoms. Like equation (36) using Wick rotation $t \rightarrow i \tau$ and assuming the new parameter (e.g. $x$ ) instead of "time", and $p_{x}$ instead of $E$, it is straight forward to conclude the relations for momentums:

$$
p_{x}=\frac{1}{2} \frac{\partial}{\partial x} \log g
$$

Comparing these equations with Hamilton-Jacobi formalism reveals the role of $-\frac{1}{2} \log g$ as the Hamilton principal function $F$. This function acts as Euclidean action $A^{E}$ with similar equations for Hamiltonian $H$ and momentum $p_{i}$ :

$$
\frac{\partial F}{\partial \tau}=\frac{\partial A^{E}}{\partial \tau}=-H \quad, \quad \frac{\partial F}{\partial q_{i}}=\frac{\partial A^{E}}{\partial q_{i}}=p_{i}
$$


This shows that $\quad A^{E}=F=-\varphi=-\frac{1}{2} \log g$

This satisfies the basic relation in BDM model i.e.:

$\begin{array}{ll}\langle E\rangle=\frac{\partial}{\partial \tau}\left(\frac{1}{2} \log g\right)=-\frac{\partial F}{\partial \tau} \\ \text { And consequently: } & p_{x}=-\frac{1}{2} \frac{\partial}{\partial x} \log g=\frac{\partial F}{\partial x}\end{array}$

Regarding (38), (39),(41) and (42) a simple solution to $g$ will be read as:

$$
\sqrt{g}=e^{(E \tau-\boldsymbol{p} . \boldsymbol{r})}
$$

Where $\boldsymbol{p}$ and $\boldsymbol{r}$ denoted as the momentum and position vectors. Therefor with the definition $A^{E}=$ $-(E \tau-\boldsymbol{p} . \boldsymbol{r}), \sqrt{g}$ takes the form:

$$
\sqrt{g}=e^{-A^{E}}
$$

Therefor the Euclidean action in BDM theory could be derived from its metric determinant.

\section{Ricci flow as a consequence of BDM model}

If $D_{v}$ denotes the binary data matrix for physical parameter $x_{v}$, then a DFT (Discrete Fourier transform) transformation of basis vectors $e_{i}^{* v}$ maps them to a set of new complex bases $\tilde{e}_{i}^{* v}$ with complex components. Discrete Fourier Transform of a binary sequence; $e_{i}^{* v}=\left\langle j_{1}^{v}, j_{2}^{v}, \ldots j_{m_{v}}^{v}\right|$ with $j_{n}^{v} \in\{\mathbf{0}, \mathbf{1}\}$ is defined as:

$$
Z_{k}^{v}(i)=\sum_{n} j_{n}^{v}(i) e^{-2 \pi j n k}
$$

So, the binary basis transform to the complex basis:

$$
j_{n}^{v} \rightarrow Z_{k}^{v}
$$

According to Parseval theorem DFT is an isometric map from the real manifold to a complex manifold with Riemannian metrics and consequently a Kahler complex manifold. This reveals that the manifold $(\mathcal{M}, g)$ is the real version of a general complex manifold and obeys the general properties of a Kahler manifold.

As we saw in previous sections, the Hamilton's principal function takes the form:

$$
F=-\frac{1}{2} \log g
$$

Consequently, we could derive the exact equations for momentum:

$$
p_{i}=-\frac{1}{2} \frac{\partial}{\partial x^{i}} \log g=\frac{\partial F}{\partial x^{i}}
$$

In the non-relativistic approach, we can choose $p_{i}=m v_{i}$, then (54) reads becomes:

Ricci tensor on a Kahler manifold reads as [ ]:

$$
m v_{i}=-\frac{1}{2} \frac{\partial}{\partial x^{i}} \log g=\frac{\partial F}{\partial x^{i}}
$$

$$
R_{i \bar{\jmath}}=\frac{\partial^{2} \log g}{\partial z_{i} \partial \bar{z}_{j}}
$$

This equation describes Ricci tensor on a Kahler manifold where we define the main manifold of BDM theory. Substitution of $\frac{\partial}{\partial z_{i}}$ and its conjugate by $\left(\frac{\partial}{\partial x_{i}}-i \frac{\partial}{\partial y_{i}}\right)$ and $\left(\frac{\partial}{\partial x_{i}}+i \frac{\partial}{\partial y_{i}}\right)$ and taking into account the independency of $g_{\mu \nu}$ and $g$ of imaginary coordinate $y_{i}$ summarizes equation of $R_{i \bar{j}}$ to:

$$
R_{i j}=\frac{\partial^{2} \log g}{\partial x_{i} \partial x_{j}}
$$

By equation (46) we obtain an interpretation for $R_{i j}$ in BDM model:

$$
R_{i j} \cong \frac{\partial p_{i}}{\partial x^{j}}=\frac{\partial p_{j}}{\partial x^{i}}
$$

Recalling the symmetry property of $R_{i j}$ as a symmetric bilinear from and $p_{i}=m v_{i}$ results in:

$$
\frac{\partial v_{i}}{\partial x^{j}}=\frac{\partial v_{j}}{\partial x^{i}}
$$


This equation means that the flow is irrotational and curl vanishes. Moreover the rate of change of particle density is proportional to $\frac{\partial v_{i}}{\partial x^{j}}$ (or $\frac{\partial v_{j}}{\partial x^{i}}$ ):

$$
\frac{\partial n_{i j}}{\partial t} \sim \frac{\partial v_{i}}{\partial x^{j}}=\frac{\partial v_{j}}{\partial x^{i}}
$$

This rate in BDM can be represented by $\dot{g}_{i j}$, therefor we have:

Finally, we get the equality:

$$
\begin{gathered}
\frac{\partial g_{i j}}{\partial t}=\frac{\partial n_{i j}}{\partial t} \sim \frac{\partial v_{i}}{\partial x^{j}}=\frac{\partial v_{j}}{\partial x^{i}} \\
\frac{\partial g_{i j}}{\partial t} \cong R_{i j}
\end{gathered}
$$

This means that Ricci flow family is compatible and a resultant flow of BDM theory.

\section{Evolution of manifold $\mathcal{M}$ under Ricci flow Dynamic}

The very first notion of Ricci flow[22, 23] which Introduced by Hamilton (1984) as an evolution equation of metric tensor:

$$
g_{i j, 0}=-2 R_{i j}
$$

Hamilton showed the unique solution of Ricci flow equation on a closed manifold for sufficiently short time. It is noteworthy that Ricci flow is an evolution equation comparable to Heat diffusion and is not a tensor equation because the derivative of metric tensor is not generally a tensor. The equation (49) shows similarities with evolution model in ADM and Numerical relativity [9]. In section (6) we have proved the Ricci flow as a direct consequence of binary matrix model. We show that this flow and its solution is in agreement with our notion of Ricci tensor and mean energy based on binary data matrix. These equations reveal straight- forward similarity between Einstein gravity emerging from curvature of space-time and curvature in data space, perhaps includes leading reasons for emerging gravity as an entropic force. Taking into account expression for $\Gamma_{0 m}^{j}$ :

$$
\Gamma_{0 m}^{j}=\frac{1}{2} g^{j k}\left(g_{k 0, m}+g_{k m, 0}-g_{0 m, k}\right)
$$

And orthogonality of time base vector (Killing vector) relative to the other bases, by $g^{m 0}=g_{k 0}=0$ for

$k, m \neq 0$ results in: $\quad H_{m j}=\Gamma_{0 m}^{j}+C_{m}^{j}=\frac{1}{2} g^{j k} g_{k m, 0}+C_{m}^{j}$

Assuming a Ricci flow like dynamic proved in section 15:

$$
R_{k m}=\alpha g_{k m, 0}
$$

With $\alpha$ as an arbitrary constant, for the sake of simplicity, we take it as $\alpha=1$. By $g_{k 0}=0$ and equation (46) we obtain:

$$
\Gamma_{0 m}^{j}=g^{j k} R_{k m}
$$

Then equations (31), (32) are converted to:

$$
\langle E\rangle=\Gamma_{0 j}^{j}=g^{j k} R_{k j}=R=\frac{1}{2} \frac{\partial \varphi}{\partial \tau}
$$

Thus gives rise to:

$$
\langle E\rangle=R
$$

Here we use the expression for trace of $\Gamma_{0 m}^{j}$, and definition for Ricci scalar curvature. As before $\varphi$ stands for the logarithm of determinant of metric tensor; $\varphi=\log g$. 
Corollary If metric tensor $g_{i j}$ for a system is defined by equation (11) then the related partition function could be derived by equation: $\quad Z=g^{-\frac{1}{2}}$

Proof: The relation for mean energy of a mixed system with density partition function $\mathbb{Z}$ in thermal equilibrium at temperature $T$, given by [24]:

$$
\langle E\rangle=U=k_{B} T^{2} \frac{\partial}{\partial T} \log \mathbb{Z}
$$

Where $k_{B}$ stands for Boltzmann constant. By replacing $T$ by $\left(k_{B} \tau\right)^{-1}$ as in thermal field theory [25] this equation transforms to:

$$
\langle E\rangle=k_{B} T^{2}\left(\frac{-1}{k_{B} T^{2}} \frac{\partial}{\partial \tau}\right) \log \mathbb{Z}=-\frac{\partial}{\partial \tau} \log \mathbb{Z}
$$

Comparing this equation with (64) results in:

Then we have:

$$
\begin{gathered}
-\frac{\partial}{\partial \tau} \log \mathbb{Z}=\frac{1}{2} \frac{\partial}{\partial \tau} \log g \\
Z=C g^{-\frac{1}{2}}
\end{gathered}
$$

Where $C$ is a constant respect to time.

\section{Least action principle and Einstein Field Equation}

In section (5) we showed the manifold $\mathcal{M}$ with a local curvature $R$ representing the mean energy per particle at any point on this manifold. The particle density $\rho_{n}$ (the number of particles per unit parameter volume at each point) evidently is not a strict function of metric $g_{\mu \nu}$ and consequently its variation respect to $g_{\mu \nu}$ vanishes. Then energy density on manifold will be denoted by $\rho_{n}\langle E\rangle$. By this energy density the variation of Euclidean action integral in our model could be read as:

$$
\delta S^{B D M}=\delta \int_{\mathrm{M}} \rho_{n}\langle E\rangle \sqrt{g} d^{n} \boldsymbol{\omega} d \tau=\delta \int_{\mathrm{M}} \rho_{n} \frac{1}{2} \frac{\partial \varphi}{\partial \tau} \sqrt{g} d^{n} \boldsymbol{\omega} d \tau=0
$$

It is straight forward to impose the relation $\delta S=0$ which guarantees the role of $\rho_{n}\langle E\rangle$ as a Hamiltonian. Subsequently the action $S$ will remain invariant under coordinate transformation. This means that the covariant divergence of $H_{\mu v}=\left(\frac{\delta S}{\delta g^{\mu \nu}}\right)$ should be vanished:

$$
H_{\mu v ; v}=\nabla_{v}\left(\frac{\delta S}{\delta g^{\mu \nu}}\right)=0
$$

This can be easily verified by taking the covariant derivative of $\frac{\delta S}{\delta g^{\mu \nu}}$ as follows. Replacing $\frac{\partial \varphi}{\partial \tau}$ by the term $\left(\frac{\partial}{\partial \tau} \log g\right)$ and some variation calculus, equation (58) gives rise to:

$$
\delta S^{B D M}=\delta \int_{\mathrm{M}} \rho_{n} \frac{1}{2} \frac{\partial \varphi}{\partial \tau} \sqrt{g} d^{n} \boldsymbol{\omega} d \tau=\frac{1}{2} \rho_{n} \int_{\mathrm{M}}\left(g_{\mu \nu, 0}-\frac{1}{2} g^{j k} g_{j k, 0} g_{\mu \nu}\right) \delta g^{\mu \nu} \sqrt{g} d^{n} \boldsymbol{\omega} d \tau=0
$$

Let $H_{\mu \nu}=\left(g_{\mu \nu, 0}-\frac{1}{2} g^{j k} g_{j k, 0} g_{\mu \nu}\right)$. Note that the density in the parameter space; $\rho_{n}$ due to Liouville theorem is independent of $g^{\mu \nu}$ and time and acts as a constant under variation. Recall the variation of Einstein-Hilbert action in $n+1$ dimension namely:

$$
\delta S^{E-H}=\delta \int_{\mathrm{M}} R \sqrt{g} d^{n} \boldsymbol{x} d \tau=\int_{\mathrm{M}}\left(R_{\mu \nu}-\frac{1}{2} R g_{\mu \nu}\right) \delta g^{\mu \nu} \sqrt{g} d^{n} x d \tau=0
$$

Where $G_{\mu \nu}=R_{\mu \nu}-\frac{1}{2} R g_{\mu \nu}$ is Einstein tensor. We have proved in the Ricci flow like dynamics as a straightforward consequence of BDM model section (6): 


$$
g_{\mu \nu, 0}=\alpha R_{\mu \nu}
$$

Substitution of $g_{\mu \nu, 0}$ by $\alpha R_{\mu \nu}$ (with $\alpha$ as a constant) in BDM action (68), results in the equation:

$$
\frac{1}{2} \rho_{n} \alpha \int_{\mathrm{M}}\left(R_{\mu \nu}-\frac{1}{2} R g_{\mu \nu}\right) \delta g^{\mu \nu} \sqrt{g} d^{n} \boldsymbol{\omega} d \tau=0
$$

Deleting the constants $\rho_{n}$ and $\alpha$ gives:

$$
\int_{\mathrm{M}}\left(R_{\mu \nu}-\frac{1}{2} R g_{\mu \nu}\right) \delta g^{\mu \nu} \sqrt{g} d^{n} \boldsymbol{\omega} d \tau=0
$$

Comparing $H_{\mu \nu}$ and $G_{\mu \nu}$ reveals that $G_{\mu \nu}=H_{\mu \nu}$ with the same solutions of equation $\delta S^{E-H}=0$ for $\delta S=0$ Thus, under the condition of Ricci flow like dynamics, two integrand remain proportional i.e. $H_{\mu \nu}=G_{\mu \nu}$ and solution of two action integral will be identical. This reveals that imposing Ricci flow as a natural consequence of BDM on evolution of manifold gives the structure of space-time in General relativity and Einstein field equations.

\section{Other Results:}

In this section we bring some examples for compatibility of the results of previous sections with some well-known results of astronomy, information theory, thermodynamics etc.

\subsection{Relation to average energy in canonical ensemble statistics}

In this section we prove an interesting relation between energy averages $\langle E\rangle$ and partition function in the path integral notion of quantum field statistics. First we note the relation of imaginary time periods $\tau=$ $-i t$ in thermal field theories which coincides the Wick rotation we used in section. 3 and $\beta=\frac{1}{k_{B} T}$ (with $k_{B}$ as Boltzmann constant) in statistical mechanics:

$$
\tau=\beta=\frac{1}{k_{B} T}
$$

Now recall the well-known derivation of average energy from canonical partition function [24].

$$
\langle E\rangle=U=-\frac{\partial \log \mathbb{Z}}{\partial \beta}
$$

According to equations (31) and (32) and assuming $\mathbb{U}_{0}=0$ :

$$
\langle E\rangle=\frac{1}{2} \frac{\partial}{\partial \tau} \log g=\frac{1}{2} \frac{\partial \varphi}{\partial \tau}
$$

Considering the relation between partition function $\mathbb{Z}$ and determinant of a non-negative self-adjoint (symmetric) operator $A$ in the context of field theory [25] gives:

$$
\mathbb{Z}=\int_{\mathrm{M}} e^{-<\varphi, A \varphi>} D \varphi=(\operatorname{det} A)^{-\frac{1}{2}}
$$

Or in a brief notation [26]:

$$
\mathbb{Z}=\int_{\mathrm{M}} e^{-\beta<E>} D \varphi
$$

Here $\langle E\rangle$ should be introduced as Dirichlet energy [26]. We see if one assumes $g_{i j}$ as an operator $A$ in above equations, then equations (66), (67) and corollary (i.e. $Z=g^{-\frac{1}{2}}$ ) yields the identity: 


$$
\langle E\rangle=\frac{\partial}{\partial \tau} \log \sqrt{g}=-\frac{\partial}{\partial \tau} \log \mathbb{Z}=-\frac{\partial}{\partial \tau} \log \int_{M} e^{-\tau<E>} D \varphi=\langle E\rangle
$$

This reveals the compatibility of expected energy formula (66) of the model with field theory formalism.

\section{$9.2 \quad$ Stress-Energy tensor}

Energy momentum tensor of model can be derived by variation of action $S$ respect to $g_{\mu \nu}$ :

Recall the Einstein field equation: $\quad R_{\mu \nu}-\frac{1}{2} g_{\mu \nu} R=\kappa T_{\mu \nu}$

$$
\begin{aligned}
& \bar{T}_{\mu \nu}=\frac{\delta S}{\delta g^{\mu \nu}}=H_{\mu \nu} \sim \frac{1}{2}\left(g_{\mu \nu, 0}-\frac{1}{2} g^{j k} g_{j k, 0} g_{\mu \nu}\right) \\
& \quad R_{\mu \nu}-\frac{1}{2} g_{\mu \nu} R=\kappa T_{\mu \nu}
\end{aligned}
$$

With assumption of Ricci flow as the governing dynamics and replacing $g_{k j, 0}=-2 R_{k j}$ in (69), we obtain the energy momentum tensor of model: $\quad \bar{T}_{\mu v}=H_{\mu \nu} \sim R_{\mu \nu}-\frac{1}{2} g_{\mu \nu} R=\kappa T_{\mu \nu}$

This is also consistent with the result of previous section and implies a linear relation between $\langle E\rangle$ and temperature.

\subsection{Energy equipartition and Landauer's principle}

As we showed in previous sections, the state of system could be characterized by a data matrix containing a set of information bits. Any column of this matrix (i.e. $\xi_{m \times 1}^{\alpha}$ ) contains the set of information of one particle in which the values of parameters given by $\mu$ bit of information, each bit for a positive interval in each degree of freedom ( $\mu$ denotes the degree of freedom). If $\bar{\epsilon}$ stands for the mean energy of a bit of information, then the mean energy per particle $\langle E\rangle$ is given by:

$$
\langle E\rangle=\mu \bar{\epsilon}
$$

Regarding the Landauer's principle which states that for erasing a bit of information the minimum required energy is: $\quad \bar{\epsilon}=k_{B} T \log 2$

Substitution of $\bar{\epsilon}$ in equation (72) gives:

$$
\langle E\rangle=\mu \bar{\epsilon}=\mu k_{B} T \log 2
$$

This is in analogy with the equipartition theorem of energy which states that the mean energy of each particle is proportional to the degree of freedom, Boltzmann constant and temperature, and shows the compatibility of the model with these two basic principles.

\subsection{Universe Inflation and extrinsic curvature}

If except for spatial dimensions all other parameters confine in a relatively equilibrium range, the large part of $g_{i j}$ will be constant with a fairly good approximation while the 3D-space metric $\boldsymbol{g}_{i j}$ increases as was proved in (35). In this situation which could be realized by our universe, the change rate of the whole metric $g_{i j}$ equals the change rate of spatial part of the metric. Therefor we can substitute the 3dimensional spatial metric $\boldsymbol{g}_{i j}$ with $j=1,2,3$ into FRW equation which reveals a relation between spatial metric tensor and scale factor $a(t)[27]$ :

$$
\boldsymbol{g}_{i j}=a^{2}(t) \delta_{i j}
$$

In $n$ dimensional manifold the determinant of above metric reads as: 
$\begin{aligned} & \boldsymbol{g} \\ & =a^{2 n}(t) \\ \text { Accordingly we have: } & \mathbb{E} \sim \frac{1}{2} \frac{\partial}{\partial \tau} \log \boldsymbol{g}=n \frac{\partial}{\partial \tau} \log a(t)=n \frac{\dot{a}}{a}\end{aligned}$

With $\dot{a}$ as time derivative of scale factor $a$ and $\mathbb{E}$ as total energy content of universe. Hubble parameter has been defined as:

Substitution in (76) gives:

$$
H=\frac{\dot{a}}{a}
$$

$$
\mathbb{E} \sim R=n H
$$

Usually Hubble parameter $H$ considered to be equal to $\mathcal{R}^{-1}$ with $\mathcal{R}$ as the observable Universe radius. Respect to the equations (87) we achieve the equation:

$$
\mathbb{E}=n \mathcal{R}^{-1}
$$

On the other hand, in ADM notion of general relativity, $3+1$ dimensional splitting of space-time reveals a relation between $K$ (the trace of extrinsic curvature $K_{i j}$ ) and determinant of metric tensor i.e. $g$ [9]:

$$
\frac{1}{2} \frac{\partial}{\partial \tau} \log \boldsymbol{g}=-\alpha K+D_{i} \beta^{i}
$$

Where $\beta^{i}$ stands for shift vector and assumed to be vanished. Therefore, by equation (86) we have:

$$
\mathbb{E}=R=-\alpha K
$$

This relation supports the curvature concept of $\langle E\rangle$ as predicted in our model. In $3+1$ decomposition of general relativity, one of the important concepts related to 3 spatial submanifold is external curvature $K_{i j}$ with a well-known equation as follows[9]:

$$
\partial_{0} g_{i j}=-2 N K_{i j}
$$

Where $N$ is considered as a constant respect to time. On the other hand, as mentioned in previous sections by applying the Ricci flow, dynamic for metric tensor reads as:

$$
\boldsymbol{g}_{i j, 0}=-2 R_{i j}
$$

This reveals that the Ricci flow can be traced out as the main dynamic in time evolution of spatial hypersurfaces in ADM formalism and Numerical relativity and supports the main idea of our model in applying this flow as a universal dynamics.

The interesting result of equation: $\quad\langle E\rangle=\frac{1}{2} \frac{\partial}{\partial \tau} \log g$ Indicates that not only the spatial coordinates, but also all other physical parameters undergo expansion of their base vectors. Linear and angular momentum subsequently obey the conventional patterns of Universe inflation. We call this fact as the generalized inflation principle and in section 9.19 show that the velocity curve problem of far stars in galaxies and dark matter could be resolved by the results of this principle. As the Hubble law holds true just for large astronomical distance at $2-4 \mathrm{Mpc}$, the generalized inflation principle applies the distances beyond these intervals.

\subsection{Equivalence of action and entropy of Black holes}

Black holes reveal an exact entropy and actions. The entropy of black holes has been widely investigated by Hawking and Beckenstein [30,31]. This entropy has been proved to be proportional to the black holes' surfaces without a statistical inference. Recent approach to Euclidean action of black holes [32] while smearing the delta function distribution to a Gaussian distribution in the limit $\sigma \rightarrow 0$ has shown an equivalence between Euclidean action and entropy of black holes.

In this section we try to show in the context of BDM model the relation between Fisher information metric and entropy and action of black holes and equivalence of gravitational action and entropy of black holes. In this approach we apply the basic results of BDM theory and to prove the equivalence of entropy and 
action of black holes. Fisher information metric matrix definition results in the equivalence of inverse of covariance matrix $\left(C^{i j}\right)$ and fisher metric tensor [34]:

$$
g_{i j}=\left(C_{i j}\right)^{-1}=C^{i j}
$$

Where $g_{i j}$ stands for Fisher information metric. For jointly normal random variables $\boldsymbol{x}_{\mathbf{1}}, \boldsymbol{x}_{\mathbf{2}}, \ldots \boldsymbol{x}_{\boldsymbol{n}}$ the entropy calculated as [34]:

$$
\mathcal{H}=\frac{1}{2} \log \Delta+k
$$

With constant $k=\log 2 \pi e$ and $\Delta=\operatorname{det} C_{i j}$. By (82) we have $\mathcal{H}=\frac{1}{2} \log g^{-1}+k$. Where $g$ stands for determinant of Fisher information metric. We show in the case of black holes at the limit $\sigma \rightarrow 0$ we can exchange the fisher metric with metric of BDM model (appendix A). Therefor substitution $\Delta=\operatorname{det} C_{i j}=g$ into equation (83) gives the entropy $\mathcal{H}$ in terms of BDM matric tensor and its determinant $g$. The Fisher metric $g_{i j}$ replaces the metric $g_{i j}$ in Equations (82) and (83) to result in:

$$
\frac{\partial}{\partial t} \mathcal{H}=-\frac{1}{2} \frac{\partial}{\partial t} \log g=-\langle E\rangle
$$

The relation of statistical entropy $\mathcal{H}=\frac{1}{2} \log \mathcal{g}^{-1}$ and the equation (40) reveals that $\mathcal{H}$ could be interpreted as Euclidean action $A^{E}$ or Hamilton principal function $F$. This connects two different concepts of statistics and Hamiltonian mechanics.

$$
\mathcal{H}=A^{E}
$$

This coincides the result of recent works that state the entropy of black hole is equal to Euclidean action i.e. $S_{b h}=A^{E}[31,32]$. Thus, we have:

$$
\frac{\partial S_{b h}}{\partial \tau}=\frac{\partial}{\partial \tau} A^{E}=-\langle E\rangle
$$

\subsection{Maxent and Least Action Principle}

The equivalence between action integral and entropy conveys the relation between least action principle and maximum entropy (Maxent) principle. If we accept the opposite signs of entropy and action integral:

$$
S=-I
$$

Where $S$ stands for entropy and $I$ for action integral. Then the maximum of action results in the minimum (extremum) of action integral: $\quad \delta S=-\delta I=0$

\section{$9.7 \quad$ Lagrangian}

Following the results of identity (40); $F=-\frac{1}{2} \log g$ we can derive the Lagrangian as:

$$
\frac{d F}{d \tau}=L=T-U
$$

For a free particle i.e. $U=0$ the expansion of (16) reads as:

For free particle $E=T$, then we have:

$$
\frac{d F}{d \tau}=\frac{\partial F}{\partial \tau}+\frac{\partial F}{\partial x} \frac{d x}{d \tau}=-E+p_{x} v_{x}=T
$$

$$
E=T=\frac{1}{2} m v_{x}^{2}
$$

Matrix version for Lagrangian could be derived by replacing $F$ by $-\frac{1}{2} \log g$ :

$$
L=\frac{d F}{d \tau}=-\frac{1}{2} \frac{d}{d \tau} \log g=-\frac{1}{2 g} \frac{d g}{d \tau}
$$

Using Jacobi formula for derivative of determinant we get: 
Taking into account $\left(g_{i j}\right)^{-1}=\frac{1}{g} C_{i j}$ we have:

$$
L=-\frac{1}{2 g} \operatorname{Tr}\left(C_{i j} \frac{d g_{i j}}{d \tau}\right)=-\frac{1}{2} \operatorname{Tr}\left(\frac{1}{g} C_{i j} \frac{d g_{i j}}{d \tau}\right)
$$

$$
L=-\frac{1}{2} \operatorname{Tr}\left[\left(g_{i j}\right)^{-1} \frac{d g_{i j}}{d \tau}\right]=-\frac{1}{2} \operatorname{Tr}\left(G^{-1} \dot{G}\right)
$$

Where $G=D D^{T}$. This is the matrix form for Lagrangian in BDM.

\subsection{Derivation of Schrodinger wave function}

If $D_{v}$ denotes the binary data matrix for physical parameter $x_{v}$, then a DFT (Discrete Fourier transform) transformation of basis vectors $e_{i}^{* v}$ maps them to a set of new complex bases $\tilde{e}_{i}^{* v}$ with complex components. Discrete Fourier Transform of a binary sequence; $e_{i}^{* v}=\left\langle j_{1}^{v}, j_{2}^{v}, \ldots j_{m_{v}}^{v}\right|$ with $j_{n}^{v} \in\{\mathbf{0}, \mathbf{1}\}$ is defined as:

$$
Z_{k}^{v}(i)=\sum_{n} j_{n}^{v}(i) e^{-2 \pi j n k}
$$

So, the binary basis transform to the complex basis:

$$
j_{n}^{v} \rightarrow Z_{k}^{v}
$$

According to Parseval theorem DFT is an isometric map from the real manifold to a complex manifold with Riemannian metrics and consequently a Kahler complex manifold. It could also be proved that these complex bases $\tilde{e}_{i}^{* v}$ are orthogonal for a specific parameter $x_{v}$ and their inner products with complex bases $\tilde{e}_{i}^{* \mu}$ of another parameter $x_{\mu}$ returns the metric tensor $g_{\mu \nu}$. So, with DFT of real bases the metric tensor will be preserved. If $\boldsymbol{c}$ denotes a matrix with the set of row matrices $\left[\tilde{e}_{i}^{* v}\right]$ while index " $i$ stands for the number of interval for a specific value of parameter $x_{v}$ assumed to be constant and $v$ denotes the number of parameter as well as the row number of $\boldsymbol{c}$, then we have:

$$
\boldsymbol{c} \boldsymbol{c}^{\dagger}=\boldsymbol{G}=\left\|g_{\mu \nu}\right\|
$$

If the determinant of $\boldsymbol{c}$ be defined by Cauchy-Binet formula, then taking the determinant of both sides of (98) gives:

$$
(\operatorname{det} \boldsymbol{c})(\operatorname{det} \boldsymbol{c})^{*}=\operatorname{det} \boldsymbol{G}=g
$$

Obviously det $\boldsymbol{c}$ is complex valued. We replace it by $\psi$. Therefor $\psi$ takes the form:

$$
\psi=\sqrt{g} e^{i \theta}
$$

Imposing the reverse Wick rotation on real time axis, results in the transformation $E \tau \rightarrow i E t$, therefor the equations (42) and (43) change to:

$$
\sqrt{\tilde{g}}=e^{i(E t-\boldsymbol{p} . \boldsymbol{r})}
$$

Complexification of the manifold and reverse Wick rotation transforms real $g$ to complex $\tilde{g}$. In $\tilde{g}$ the new time parameter was added with $v=0$ and this results in additional negative and imaginary entries to $\boldsymbol{G}$ to form complex $\widetilde{\boldsymbol{G}}$ with complex determinant $\tilde{g}$.

If action denoted by $S$, the new action will be $\tilde{S}=i S$ so we obtain:

$$
\begin{array}{lc} 
& -i S=\log \sqrt{\tilde{g}} \\
\text { Substitution } \tilde{g} \text { in Equation (99) gives: } & \sqrt{\tilde{g}}=\psi e^{-i \theta} \\
\text { Inserting (101) into (100): } & -i S=\log \left(\psi e^{-i \theta}\right) \\
\text { And finally: } & \psi=e^{-i(S-\theta)}=\alpha e^{-i S}
\end{array}
$$

Where $\alpha$ stands for a complex constant. The relation $\psi \sim \alpha e^{-i S}$ guarantees the Schrodinger equation:

$$
i \frac{\partial \psi}{\partial t}=\Delta \psi
$$

$\psi$ is the determinant of matrix $\boldsymbol{c}$ and takes the multilinear form of $\boldsymbol{c}$ entries. Using the general definition of determinant in the context of Cauchy-Binet formula, the determinant is a multilinear form of $\boldsymbol{c}$. It is straight forward to impose the time partial derivatives on this summation and deduce that Schrodinger equation (103) holds for all entries of $\boldsymbol{c}$. Then for each particle we have a similar equation: 


$$
i \frac{\partial \psi_{n}}{\partial t}=\Delta \psi_{n}
$$

Where $\psi_{n}$ stands for a function of parameters for $n$th particle. At any point in the parameter space it turns out the corresponding entries of $n$th particle for specified parameter.

\subsection{Derivation of Slater Determinant}

Consider sub-matrix $D_{v}$ of $D$ which contains the coordinate $v$ of related parameter. If all particles set to be fermions, Pauli Exclusion Principle limits the number of particles in each row by 1 . This means that the related matrix $D_{v}$ is of order $N \times N$ and its determinant takes the values 1 or -1 . DFT transformation of $D_{v}$ changes the real basis to complex basis and produces a complex manifold with Riemannian metrics. Consequently, this manifold is compatible with a Kahler manifold. Let denote the transformed $D_{v}$ by $\widetilde{D}_{v}$. If we restrict the notation for $\widetilde{D}_{v}$ to a spatial parameter $x$ with other parameters independent of time, then $\widetilde{D}_{x}$ contains the entries defined by $\psi_{n}(x)$ as proved in (30). Therefor if we would define $\Phi$ as the determinant of $\widetilde{D}_{x}=\psi_{n}(x)$, the determinant of corresponding block matrix $G_{x}$ in $G$ appears as:

Thus we have:

$$
\begin{aligned}
& g_{x}=\left[\operatorname{det} \psi_{n}(x)\right]\left[\operatorname{det} \psi_{n}(x)\right]^{*} \\
& \Phi=\left[\operatorname{det} \psi_{n}(x)\right] \sim \sqrt{g_{x}} \sim e^{-i S}
\end{aligned}
$$

This means that $\Phi$ is the wave function of $N$ fermions as known as the Slater determinant.

\section{$9.10 \quad$ Ideal gas}

Ideal gas as a non-interacting system of particles brings a good example for our model. Let us consider a confined ideal gas with volume $V_{1}$ while imposing a compression contracted to volume $V_{2}$ with change of energy $\Delta E=W$, where $W$ denotes the performed work. If the contraction coefficient defined as $\alpha=\frac{V_{1}}{V_{2}}$ , and contraction occur along $x$ axis, then the related BDM metric transforms by scale factor $\alpha$ on the basis $e_{i}^{* v}$ which corresponds to $x$ axis. Evidently the reason is the increase of particle number density along $x$ axis proportional to $\alpha$. Since the entries of $g_{i j}$ are the inner product of basis vectors $e_{i}^{* v}$, the resultant change in determinant of $g_{i j}$ will be the product by scale factor $\alpha^{2}$. Consequently the $\sqrt{g}$ changes to $\sqrt{g^{\prime}}=\alpha \sqrt{g}$. Therefor taking the action $S=-\log \sqrt{g}=E t$, the energy change reads as:

$$
\Delta E . t=\log \frac{\sqrt{g^{\prime}}}{\sqrt{g}}=\log \alpha=\log \frac{V_{1}}{V_{2}}
$$

Taking into account the periodic time and replacing time with $1 / k T$, we have:

$$
\Delta E=k T \log \frac{V_{1}}{V_{2}}
$$

Which is compatible with the formulas of ideal gases.

\subsection{Symmetries of BDM theory}

BDM model is compatible with a few symmetries. Here we list the most important symmetries a

1) Unitary symmetries: we have shown that DFT transform of data matrix $D$ leaves the determinant $g$ invariant. Similarly, all unitary transformations are symmetric groups of BDM model. Let $U$ denotes an arbitrary unitary matrix which transforms the data matrix $D$ :

$$
D^{\prime}=U D
$$

Taking the determinant:

$$
\operatorname{det} G^{\prime}=\operatorname{det} D^{\prime} D^{\prime \dagger}=\operatorname{det} U D \operatorname{det}(U D)^{\dagger}=\operatorname{det} U \operatorname{det} U^{\dagger} \operatorname{det} D D^{\dagger}=\operatorname{det} G
$$

This reveals the unitary symmetries of Lagrangian in BDM model. 
2) Conformal symmetries: a conformal symmetry refers to those transformation of metric tensors that rescales the metric either global or local while the theory remains invariant:

$$
g_{\mu \nu} \rightarrow \Omega^{2} g_{\mu \nu}
$$

Where $\Omega^{2}$ stands for a function of space time coordinates.

Determinants of metric transform as:

If $\Omega^{2}$ is time independent then we obtain:

$$
g^{\prime}=\Omega^{2 n} g
$$

For time dependent $\Omega^{2}$ we get:

$$
L^{\prime}=-\frac{d}{d \tau} \log \sqrt{g}{ }^{\prime}=-\frac{d}{d \tau}(\log \sqrt{g}+n \log \Omega)=L
$$

$$
L^{\prime}=L-n \frac{d}{d \tau} \log \Omega
$$

Equation (113) guarantees the invariance of Lagrangian under conformal transformation, because additional term is a total time derivative. Therefor the conformal symmetry is an inherent symmetry of BDM theory.

\subsection{Bremermann limit and maximum Curvature}

In this section we reveal an interesting relation between Bremermann limit and maximum space-time curvature and Plank time and length. Bremermann limit is the upper bound of bit information transfer rate per unit mass and is defines as $\frac{c^{2}}{\hbar}$. Due to the dynamic of Ricci flow we have:

$$
R_{\mu \nu}=\frac{1}{2} \dot{g}_{\mu \nu}
$$

For a space with spherical symmetry (like the black holes) Ricci tensor equals extrinsic curvature i.e.

$$
R_{\mu \nu} \cong K_{\mu \nu}
$$

The scaler curvature for 3-hyper surface with spherical symmetry reads as:

$$
R \cong \frac{1}{r^{3}}
$$

To be compatible with physical scaler curvature it requires a Planck constant $\hbar$ to admit the correct dimension of $R$ :

$$
R=\frac{\hbar}{r^{3}}
$$

Now respect to the limit of maximum bit information rate (i.e. maximum average of transmitted bit per second) known as Bremermann limit (we denote it by $L_{B}$ ), we should consider the maximum limit of $\dot{g}_{\mu \nu}$ as Bremermann limit. The main reason is the structure of $g_{\mu \nu}$ in BDM theory. In BDM the entries of $g_{\mu \nu}$ is the time average of number of bits restricted to some intervals of physical variables. Therefor the maximum changing rate of the bits involved in $g_{\mu \nu}$ i.e. $\dot{g}_{\mu \nu}$ will be proportional to Bremermann limit:

$$
\left(\dot{g}_{\mu \nu}\right)_{\max } \sim L_{B}
$$

To satisfy homogeneity of dimensional equation, respect to Einstein field equation dimensional homogeneity: $\quad R_{\mu \nu}-\frac{1}{2} R g_{\mu \nu}=8 \pi G T_{\mu \nu}$

The term $g_{\mu \nu} / G$ is Dimensionless quantity required for dimensional homogeneity of equation (118). Then we should replace (117) with:

$$
C_{\max }=\frac{1}{G}\left(\dot{g}_{\mu \nu}\right)_{\max } \sim \frac{1}{G} L_{B}
$$

Where $C_{\text {max }}$ denoted as the possible maximum rate of bit (particles) transfer in BDM theory. For calculating the scaler curvature, we contract the Ricci flow (49) with $g^{\mu \nu}$ :

$$
R=g^{\mu \nu} R_{\mu \nu}=\frac{1}{2} g^{\mu \nu} \dot{g}_{\mu \nu}=\frac{1}{2} \operatorname{Tr}\left(G^{-1} \dot{G}\right)=\frac{1}{2} \frac{\partial}{\partial t} \log (\operatorname{det} G)=\frac{1}{2} \frac{\partial}{\partial t} \log g=\langle E\rangle
$$

Thus, the total scaler curvature equals the energy per particle multiplied by particle density. In each row of data matrix $D$ the number of positive bits ' 1 ' is the same as the number of particles with the same 
value of specific physical variable. Therefor the maximum energy of all bits (particles) in this interval could be calculated by multiplying the maximum energy of each particle $\varepsilon_{\max }$ by the maximum change of the number of bits (particles) in this interval i.e. $\left(\dot{g}_{\mu \nu}\right)_{\max }=L_{B}$. Then we have:

$$
E_{\max }=\varepsilon_{\max } C_{\max }
$$

Respect to (120) the maximum total curvature of involved particles is $R_{\max }=E_{\max }$. On the other hand the maximum energy per particle, due to uncertainty principle, could be derived by:

$$
\varepsilon_{\max }=\frac{\hbar}{\tau_{p}}
$$

Where $\tau_{p}$ stands for the Planck time. The relation between maximum curvature, $E_{\max }$ and equation (116) shows that $R_{\max }$ for a 3-hypersurfac with spherical symmetry is proportional to $\frac{\hbar}{r^{3}}$ when $r^{3}$ takes the minimum length $l_{p}$ (Planck length):

Equations (121), (122), and (123) results in:

$$
R_{\max }=\frac{\hbar}{l_{p}^{3}}=E_{\max }
$$

$$
\frac{\hbar}{l_{p}^{3}}=\frac{\hbar}{\tau_{p}} C_{\max }=\frac{\hbar}{\tau_{p}} \frac{L_{B}}{G}
$$

We can verify this equation by substituting the values of Planck time and length and Bremermann limit:

$$
\frac{c^{\frac{9}{2}}}{G^{\frac{3}{2}} \hbar^{\frac{3}{2}}}=\frac{c^{\frac{9}{2}}}{G^{\frac{3}{2}} \hbar^{\frac{3}{2}}}
$$

This interesting result confirms the "information bit" nature of space metrics and brings an example to the authentication of Ricci flow and compatibility of BDM theory with Planck length and time and Bremermann limit.

\subsection{Energy density of Light wave propagation}

As an example for applying the Ricci flow as the dominant dynamics of isolated free system of particles, we imagine a spherical wave front of light propagating from an origin point of space-time. Due to (120) we have:

$\langle E\rangle=R$

Therefor for energy density of propagating wave of which is an example of an isolated free system of photons we apply this equation and deduce that it equals the curvature of the system. In this example this curvature reduces to 2-dimensional sphere curvature (wave front is spherical) and amounts:

$$
\langle E\rangle=R \sim \frac{1}{r^{2}}
$$

This results explains the inverse square law for energy density of light propagation in vacuum.

\section{$9.14 \quad$ Power spectrum}

it is valuable to calculate power spectrum of a system via the BDM model. First we note that strict definition of power spectrum reads as [34]:

$$
S(\omega)=\iint_{-\infty}^{+\infty} x_{i} x_{j} G\left(x_{i}, x_{j}, \omega\right) d x_{i} d x_{j}
$$

Where $G$ stands for the Fourier transform of joint probability density $f\left(x_{i}, x_{j}, t\right)$ :

$$
G\left(x_{i}, x_{j}, \omega\right)=\int f\left(x_{i}, x_{j}, t\right) e^{-i \omega t} d t
$$

In BDM model the joint density of pair variables $x_{i}, x_{j}$ determined by $g_{i j}$ as mentioned in previous sections. Therefor the power spectrum in BDM sense reads as:

\subsection{Bit density principle}

$$
S(\omega)=\iint_{-\infty}^{+\infty} x_{i} x_{j}\left(\int g_{i j}\left(x_{i}, x_{j}, t\right) e^{-i \omega t} d t\right) d x_{i} d x_{j}
$$


In this section we show the density of bits along spatial or time coordinate, represents hidden momentum and energy of a system of particles. As we have shown in previous sections, the expression $\frac{1}{2} \log g$ could be interpreted as "entropy" in some limiting situations such as black holes. If we agree on the classic value of bit information as the entropy of a system, then we have the relation:

$$
I=-S=\frac{1}{2} \log g
$$

With this identity, the expressions for energy and momentum in BDM theory will be read as:

$$
\langle E\rangle=\frac{1}{2} \frac{\partial}{\partial t} \log g=\frac{\partial I}{\partial t} \quad, \quad p_{i}=-\frac{1}{2} \frac{\partial}{\partial x^{i}} \log g=-\frac{\partial I}{\partial x^{i}}
$$

These equations imply that there are relations between energy and momentum and bit density over time and spatial dimensions. These particle densities in BDM model indicate the bits density principle. As a generalization, the bits density over time should correspond to energy and the bits density along the angular variables correlates with angular momentum.

$$
\text { energy } \sim \frac{\text { bits }}{\text { second }}, \text { momentum } \sim \frac{\text { bits }}{\text { unit length }} \text {, angular momentum } \sim \frac{\text { bits }}{\text { unit angle }}
$$

The best example for time density of bits is the density of a full wavelength (as a single bit) over the time. The bits density over time, normally can be explained by the frequency of the propagating wave. Hence the energy should be proportional to frequency:

$$
\varepsilon \cong f
$$

For dimensional compatibility, we use $h$ as an appropriate coefficient and hence we have:

$$
\varepsilon=h f
$$

We know for electromagnetic waves (photons) this is the Planck formula for energy per photon.

For bits density along spatial coordinates this full wavelength (bit) density could be represented as $\frac{1}{\lambda}$ and therefore the momentum is proportional to $\frac{1}{\lambda}$ and after multiplying with $h$ as proportional coefficient gives the De Broglie equation for matter wave:

\subsection{Crystallography}

$$
p=\frac{h}{\lambda}
$$

BDM theory could be applied in special mode to solid state theory of crystals with its Bravais and reciprocal lattice and coordinates. In other words, crystallography theory can be considered as a special case of BDM model. We show the dual basis $e^{* v}$ in BDM model corresponds the reciprocal basis in crystallography. Metric tensor in BDM model derived from dual basis $e^{* v}$. Respect to equation (7) for $i=j$ we have:

$$
\left\langle e^{* v}(i), e^{* v}(i)\right\rangle=\sum_{n}\left[e^{* v}(i)\right]_{n}\left[e^{* v}(i)\right]_{n}^{T}=\left|e^{* v}(i)\right|^{2}=g_{v}^{v}(i)=f_{v}^{v}(i)
$$

This means that the bit density on a specific value of parameter $v$, equals the square of $e^{* v}(i)$ module. In crystallography theory the main basis is the lattice basis namely Bravais lattice. These vectors simply connect two adjacent atoms and make a 3-dimensional parallelepiped structure as the spatial base vectors. As has been mentioned before the reciprocal basis of a crystal determines the actual density of particles along axis perpendicular to the related crystal surface. Dual basis in BDM model looks like the reciprocal basis in crystal lattices. Therefore, dual basis in BDM model seems to take the momentum values and could be assigned by momentum like variables just similar to pseudo-momentum components of the Reciprocal basis of crystal lattices. consequently, there is a connection between dual bases in BDM model which reflects the probability density of particles in our system and pseudo-momentum of crystal lattices. For spatial components these dual bases stand for the real pseudo-momentum or K-vectors of crystal lattices. For bit density along the spatial coordinates in crystal lattices, the total density of a plane of atoms along the axis perpendicular to that plane, is proportional to $\frac{1}{d}$ where $d$ is the distance between atoms planes. The magnitude of corresponding reciprocal base lattice is also $\frac{1}{d}$ : 


$$
|G|=\frac{1}{d}
$$

The vector with this magnitude perpendicular to the atoms plane is called crystal momentum or pseudomomentum. This momentum appears just in the interactions of atoms lattice with an incident photon or particle waves. Respect to the bit density principle from previous section, this pseudo-momentum is equal to density of bits (atoms) over the interval $d$ by the equation:

$$
\frac{\text { bits }}{\text { unit length }}=\frac{n}{d}
$$

The related momentum is the product of density and $\hbar$ i.e.

then the pseudo-momentum per atom reads as:

$$
P=\hbar \frac{n}{d}
$$

$$
P=\hbar \frac{1}{d}=\hbar G
$$

this is the main relation for crystal momentum which derived by the bit information principles.

For non-spatial coordinates, these bases show similar concept.

Curiously, the similar relation should govern the angular momentum. The suggested relation is as follows:

$$
L_{\theta}=\frac{\hbar}{\theta}
$$

Interpretation of $\theta$ as unit angle (angular period) needs more explanation. For crystals with $N$-fold rotational symmetries it has been verified that the difference of pseudo-angular momentum of incident and diffracted photons on a crystal with $N$-fold rotational symmetries obey the relation [33]:

$$
\Delta m \hbar=\sigma \hbar+N P \hbar
$$

And for Rayleigh scattering with $\sigma_{i}$ and $\sigma_{s}$ as incident and scattered helicity of photons we have [33]:

$$
\sigma_{i}-\sigma_{s}=N P
$$

Where $P$ denotes an integer and $N$ determines the particles or bits density per unit angle $2 \pi$ (recall the definition of $N$-fold rotational symmetries). Thus the equation (134) in BDM context is compatible with pseudo-angular momentum relation in (135).

Another crystallographic evidence backing BDM model pertains to metric attributed to crystal lattice. The relation between displacements $\varepsilon_{j}=u_{j j}$ ( $u_{j j}$ denotes the diagonal strain tensor) under propagation of a single pulse wave and the metric attributed to a crystal lattice is in the form:

$$
d l^{2}=\sum_{j}\left(1+2 u_{j j}\right) d x_{j}^{2}
$$

The determinant of this metric tensor will read as:

$$
\operatorname{det} G=\prod_{j}\left(1+2 u_{j j}\right)
$$

Its logarithm for small valued $u_{j j}$ takes the form:

$$
\log \operatorname{det} G=\sum_{j} \log \left(1+2 u_{j j}\right)=\sum_{j} 2 u_{j j}
$$

then $\langle E\rangle=\frac{1}{2} \frac{\partial}{\partial \tau} \log g$ approximated by:

$$
\langle E\rangle=\sum_{j} \frac{\partial}{\partial \tau} u_{j j}
$$

If assume the displacements $\varepsilon_{j}=u_{j j}$ a periodic function of time to impose vibrations on crystal atoms, we get:

$\varepsilon_{j} \cong \varepsilon e^{i \omega t}$
Then for energy with $\tau=i t$ we have: $\langle E\rangle \cong \varepsilon \sum_{j} \frac{\partial}{\partial \tau} e^{i \omega_{j} t} \cong-i \sum_{j} i \omega_{j} e^{i \omega_{j} t}=\sum_{j} \omega_{j} e^{i \omega_{j} t}$

Considering the real part of equation, expected energy for a pulse wave through crystal wave will read as:

And for each degree of freedom: $\quad\left\langle E_{j}\right\rangle \cong \omega_{j} \int_{0}^{\frac{\pi}{2}}\left|\operatorname{co} \omega_{j} t\right|=\omega_{j}$

This means the proportionality of energy per particle and frequency. After multiplying with $\hbar$, this is compatible with Planck equation:

$$
\left\langle E_{j}\right\rangle=\hbar \omega_{j}
$$


And shows the energy of a phonon in crystal lattice.

\subsection{Derivation of mass-energy equivalence}

What is the relation of energy for a body with mass $m$ in the notion of bit information density formalism? For a body with mass $m$ the number of bits is obscure, because the constituent particles are different in mass and type and could not be assumed as individual bits in the context of BDM theory. Nevertheless, a direct approach is to define a fundamental mass unit that scales the body mass in dimensionless numbers as the information bit content. The most fundamental mass unit is presented as Planck mass by definition:

$$
m_{p}=\sqrt{\frac{\hbar c}{G}}
$$

This mass is also the minimum possible mass of a black hole. Hence the number of bits of a body with mass $m$ reads as:

$$
n=\frac{m}{m_{p}}=m \sqrt{\frac{G}{\hbar c}}
$$

due to bit density principle the related energy could be derived by calculation of bit density over a definite time interval, which is defined by Planck time:

$$
t_{p}=\sqrt{\frac{\hbar G}{c^{5}}}
$$

Then for equivalent hidden energy for mass $m$ we should work out the density of bit information over

$$
\text { time scale } t_{p} \text { times } \hbar \text { : } \quad E=\hbar \frac{n}{t_{p}}=m c^{2}
$$

This reveals the exact mass-energy formula in the context of especial relativity and asserts it as a potential form of energy that can be appeared just in the interactions with other bodies. This is equivalent with the pseudo-momentum in crystal lattices as the hidden momentum that appears when the lattice exposed to interactions with particle waves and photons.

\subsection{Beckenstein Bound}

The maximum information confined in a region with radius $R$ and energy $E$ due to Beckenstein is:

$$
I \leq \frac{2 \pi R E}{\hbar c \ln 2}
$$

Here the time span for bit information is the interval by which the light travels from center of black hole (singularity point) to horizon at the radius $R$ :

$$
\Delta t=\frac{R}{c}
$$

Therefor the bit density over this interval is proportional to energy $E$ up

$$
\text { to a coefficient } \hbar \text { : } \quad E \simeq \hbar \frac{I}{\Delta t}=\hbar \frac{I c}{R} \Rightarrow I \simeq \frac{R E}{\hbar c}
$$

is compatible with Beckenstein bound.

\subsection{Rotation (velocity) curve problem as the results of generalized inflation}

Velocity (rotation) curves of distant stars relative to their galaxy's center do not obey the Newtonian dynamics. Based on astronomical observations, the orbiting velocities of these stars are approximately equal or slowly increasing over the large distances whereas the Newtonian dynamic predicts a lowering speed proportional to $\frac{1}{\sqrt{r}}$ with $r$ as the distance of star from galaxy's center. The problem has been justified by assuming an unobservable dark matter distributed in the galaxies with a certain pattern. The modified Newtonian dynamics (MOND) is also another approach to this discrepancy by suggesting a modification of Newtonian dynamics at very large distances and very low gravity accelerations. I suggest 
an interpretation of both Universe inflation and velocity (rotation) curve in the context of BDM theory. From the main equations of the model we conclude that all base vectors of all parameters inflate through time:

$$
\langle E\rangle=\frac{1}{2} \frac{\partial}{\partial \tau} \log g
$$

Because $g$ is the determinant of parametric space metric which include base vectors of all parameters i.e. spatial, linear momentum and angular momentum. Therefor in BDM the inflation involves all parameter and not only the spatial dimension and consequently it implies a generalized inflation. The rate of inflation is proportional to energy content of the system. For universe, the expansion rate is proportional to its energy or mass content. If $a$ stands for the scale factor of this inflation, then the ratio $\frac{\dot{a}}{a}$ equals the Hubble constant $H$ :

$$
H=\frac{\dot{a}}{a}
$$

As a generalization to inflation of spatial metric, equation (151) guarantees the inflation of all parameters with equal footing. Therefore, as the Hubble law describes the expansion rate through such an equation:

$$
H=\frac{\dot{a}}{a}=\frac{\dot{r}}{r}
$$

Due to generalized inflation principle, we could also apply the same equation for linear and angular momentum:

$$
H=\frac{\dot{p}}{p}=\frac{\dot{L}}{L}
$$

For linear momentum we obtain: $\quad \dot{p}=p H \rightarrow \dot{v}=v H \rightarrow v=r H \rightarrow r \cong r_{0} e^{H t}$

Which is compatible with Hubble law. For angular momentum we have:

Or:

$$
\begin{gathered}
\dot{L}=L H \rightarrow \frac{d(v r)}{d t}=v r H \rightarrow \log v r=H t \rightarrow v r=v_{0} r_{0} e^{H t}=v_{0} r \\
v r=v_{0} r \rightarrow v=v_{0}
\end{gathered}
$$

Here $v$ stands for orbiting velocity of stars and $v_{0}$ is a constant velocity. These equations are valid at the limit of a far distance $D_{0}$ from other galaxies where the gravitational acceleration amounts to negligible limit and the observable inflation begins. At this critical distance which is about 2-4 Mpc (Mega Parsec) the radial velocity respect to Hubble's law start to be observable and yields:

$$
v_{0}=D_{0} H
$$

The initial velocity $v_{0}$ should be interpreted as the escape velocity which determines the lower limit of expansion velocity. Consequently, the orbit velocity $v$ also equals this escape velocity and obeys the expansion effects in the negligible gravity. Then we have:

$$
v=v_{0}=D_{0} H
$$

Evidently the observable expansion of galaxies is possible for galaxy's stars at very large distances where the gravity loses required binding potential to hinder expansion of spatial and non-spatial parameters. Based on astronomical observations, the least distance $D_{0}$ is about 2-4 Mpc (Mega Parsec) [38]. Then the estimated constant velocity $v_{0}$ will be read as (with $H \cong 70 \mathrm{~km} / \mathrm{s}$. Mparsec:

$$
v_{0} \cong D_{0} H=140-280 \mathrm{~km} / \mathrm{s}
$$

Surprisingly, this is the range of asymptotic constant velocities of the stars at the rim of galaxies. The acceleration emerged from generalized inflation at large distances will read as:

$$
a=\frac{v_{0}^{2}}{r}=\frac{\left(D_{0} H\right)^{2}}{r}
$$

This acceleration replaces the Newtonian acceleration around the distance $D_{0}$ and dominates it at the astronomical distances comparable to it. Therefore, we get two separable acceleration, Newtonian which is proportional to $\frac{1}{r^{2}}$ and BDM derived acceleration that is proportional to $\frac{1}{r}$. At the astronomical distances the Newtonian acceleration decreases rapidly and $v$ approaches to $D_{0} H$ as the final constant velocity that corresponds to $v_{\infty}$ in MOND theory. Equation that fits almost all observed data of galaxies rotation curves could be extrapolated in a way followed by MOND approach to connect these limits of 
velocities at the far and near astronomical distances. At this level it is compatible with MOND results after clarifying the reason for Newtonian modification at very large distances based on BDM theory results. This reveals the reason for rotation curve discrepancies without invoking the existence of hidden Dark matter or phenomenological modification of Newtonian dynamics (MOND). For far orbiting stars in a galaxy, the angular momentum obeys the Newtonian dynamics up to a distance where the expansion of parameters overcomes the gravitational field acceleration. Of course, this velocity is equal to asymptotic velocity of stars in the galaxy disk as assumed in the context of MOND theory and be achieved by stars when the distances are about $D_{0}=2-4 \mathrm{Mpc}$ from galaxy center. Stars speed behave asymptotically between the points where we arrive the fundamental acceleration $a_{0}$ and the point of critical distance $D_{0}$ which have been observed through astronomical data. Here we proved the relation between the Hubble constant, orbit velocity limit and $D_{0}$ as the minimum distance where the inflation begins.

\subsection{Physical constants and generalized inflation}

The spin of particles as an internal angular momentum with its involvement in electromagnetic interaction between electron and photon, is included in the parametric space. The any generalized inflation that evolves the angular momentum as described in previous section, would impact on electron spin. The electron spin $S$ with the value of $\frac{\hbar}{2}$ will lose its quantity due to the equation (155):

$$
S=S_{0} e^{H t}
$$

If we calculate the relative change of spin over 1 year, we get:

$$
\frac{\dot{s}}{s} \cong 6 \times 10^{-11}
$$

Fine structure constant $(\alpha)$ is among the most suspicious constant which should be evolved over universe evolution. The definition for this electromagnetic coupling constant is:

$$
\alpha=\frac{e^{2}}{\hbar c}
$$

$e$ and $c$ are constants that are not included in parameters of BDM and therefor are not involved by inflation evolution. However, $\hbar$ as an angular momentum will alter over inflation and results in negative relative change to $\alpha$. Consequently, the rate of relative change of $\alpha$ reads as:

$$
\frac{\dot{\alpha}}{\alpha}=\frac{\dot{s}}{S} \cong 6 \times 10^{-11}
$$

This change rate is compatible with one of the related data was given by Shylakhter [ 35] and reviewed by Cardenas [36,37] which is reported as $4 \times 10^{-11}$. This shows an amazing compatibility of BDM generalized inflation results and astronomical results of physical constants evolution.

\section{Appendix A}

In the case of black holes where all the physical variables of its constituent confined to infinitesimal intervals $\delta x^{i}$, around the singularity point, if we fix the center of mass of black hole on the origin of spatial coordinates, the expected values of position and momentum of constituents are near zero corresponding BDM metric will be concentrated over these intervals with negligible values out of $\delta x^{i}$ and mean values near to zero. Thus the correlation (covariance) matrix element $\mathfrak{R}_{i j}$ while the mean of all random variables vanishes i.e. $\bar{x}=0$ reads as:

$$
\mathfrak{R}_{i j}=\sigma_{i j}=\left\langle g_{i j} \delta x_{i} \delta x_{j}\right\rangle=g_{i j} \delta x_{i} \delta x_{j}
$$

Determinant of $\sigma_{i j}$ matrix (denoted by $\Delta$ ) could be calculated as:

Substitution of $\sigma_{i j}$ with $g_{i j} \delta x_{i} \delta x_{j}$ gives rise to:

$$
\Delta=\sum_{i j k \ldots} \varepsilon_{i j k \ldots} \sigma_{i 1} \sigma_{j 2} \sigma_{k 3} \ldots
$$


Logarithm of both sides results in:

$$
\Delta=g \prod_{i}\left(\delta x_{i}\right)^{2}
$$

$$
\log \Delta=\log g+2 \sum_{i} \log \delta x_{i}=\log g+C
$$

On the other hand a multivariate normal distribution at the limits $\sigma_{i} \rightarrow 0$ approaches to Dirac delta. At this limit $d x$ is proportional to $\sigma$ i.e. $\sigma_{i}=\alpha x_{i}$. This results in an equivalence between Fisher metric and space metric:

$$
g_{i j}=\left\langle\frac{\partial \log P}{\partial \theta_{i}} \frac{\partial \log P}{\partial \theta_{j}}\right\rangle \cong \frac{1}{\alpha^{2}}\left\langle\frac{\partial \log P}{\partial x_{i}} \frac{\partial \log P}{\partial x_{j}}\right\rangle \cong \frac{1}{\alpha^{2}} g_{i j}
$$

Where $\theta_{i}=\sigma_{i}$ and $P$ multivariate normal distribution. The reason is the exchangeability of $\sigma_{i}$ and $x_{i}$ in $\log P$ :

$$
\log P \cong \frac{x^{2}}{\sigma^{2}}
$$

Metric tensor $g_{i j}$ stands for Fisher metric for coordinates $x_{i}$ and could be regarded as metric of BDM theory that in empty space converts to usual metric of space and in the presence of matter requires stress - energy tensor. Therefor the limit identity (A1) reveals the equivalence of BDM entropy and Fisher entropy:

$$
\mathcal{H}=\frac{1}{2} \log \Delta=\frac{1}{2} \log g+C
$$

The distributions of $\sigma_{i}$ and $x_{i}$ from which we derive the expectations in (A1) are identical because the normal distributions at the limit $\sigma_{i} \rightarrow 0$ tends to Dirac delta:

$$
P \rightarrow \delta\left(x_{i}\right)=\delta\left(\sigma_{i} / \alpha\right)=\alpha \delta\left(\sigma_{i}\right)
$$

\section{Conclusion}

Binary data matrix (BDM) and constructed Hilbert space fitted for physical measurements recording, represents a set of base vectors with associated metric tensor and entries that interpreted as observed joint density probabilities of related system parameters. Both metric tensor and joint probabilities are symmetric and positive semi-definite. Definition of density matrix in the sense of quantum statistics conveys the full analogy between these matrices and metric tensor. We define a manifold with the dimension of the whole parameters intervals number, and its submanifolds expanded by basis vector subsets identified on each independent parameter intervals. We prove that this geometry and induced metric not only reveals the properties of a Riemannian manifold, but also proves the equivalence of metric compatibility and Liouville-Von Neumann equation. We generalize the equivalence to spatial dimensions and prove the compatibility of BDM model with Hamilton-Jacobi formalism. This model also explores the relation of the manifold curvature and ensemble average energy of the under measurement system and uncovers the rational for equating the pure geometrical side and stress energy tensor of Einstein field equation. We have shown that this mean energy is proportional to energy per bit of information that recovered from measurements. We assert the compatibility of normalized Ricci flow dynamics with our Hamiltonian action integral and equivalence of this integral with Einstein-Hilbert action integral. Other successful interpretations included in this model, consist of equipartition theorem of energy, average energy in canonical ensemble, and Landauer's principle. Compatibility with Universe inflation and FRW equation is mentioned in last section. Interestingly the concept of generalized inflation justifies the rotation curves of galaxies' far stars and obviates the assumption of dark matter with a strong theoretical framework that could substitutes the MOND theory. 


\section{References}

[1] H. Goldstein, Classical mechanics: Pearson Education India, 1965.

[2] J. J. Sakurai and J. Napolitano, Modern quantum mechanics: Addison-Wesley, 2011.

[3] W. De Muynck, W. De Baere, and H. Martens, "Interpretations of quantum mechanics, joint measurement of incompatible observables, and counterfactual definiteness," Foundations of Physics, vol. 24, pp. 1589-1664, 1994.

[4] C. Branciard, "Error-tradeoff and error-disturbance relations for incompatible quantum measurements," Proceedings of the National Academy of Sciences, vol. 110, pp. 6742-6747, 2013.

[5] M. Ringbauer, D. N. Biggerstaff, M. A. Broome, A. Fedrizzi, C. Branciard, and A. G. White, "Experimental joint quantum measurements with minimum uncertainty," Physical review letters, vol. 112, p. 020401, 2014.

[6] R. L. Hudson, "When is the Wigner quasi-probability density non-negative?," Reports on Mathematical Physics, vol. 6, pp. 249-252, 1974.

[7] R. M. Wald, General relativity: University of Chicago press, 2010.

[8] A. I. Borisenko, Vector and tensor analysis with applications: Courier Corporation, 1979.

[9] T. W. Baumgarte and S. L. Shapiro, Numerical relativity: solving Einstein's equations on the computer: Cambridge University Press, 2010.

[10] W. Rindler, Relativity: special, general, and cosmological: Oxford University Press on Demand, 2006.

[11] E. Verlinde, "On the Origin of Gravity and the Laws of Newton," Journal of High Energy Physics, vol. 2011, pp. 1-27, 2011.

[12] R. Yang, "Is gravity entropic force?," Entropy, vol. 16, pp. 4483-4488, 2014.

[13] G. Ruppeiner, "Thermodynamics: A Riemannian geometric model," Physical Review A, vol. 20, p. 1608, 1979.

[14] G. E. Crooks, "Measuring thermodynamic length," Physical Review Letters, vol. 99, p. 100602, 2007.

[15] T. Padmanabhan, "Thermodynamical aspects of gravity: new insights," Reports on Progress in Physics, vol. 73, p. 046901, 2010.

[16] S. T. Yau, "On the ricci curvature of a compact kähler manifold and the complex monge-ampére equation, I," Communications on pure and applied mathematics, vol. 31, pp. 339-411, 1978.

[17] M. A. Nielsen and I. L. Chuang, Quantum computation and quantum information: Cambridge university press, 2010.

[18] M. Ohya and I. Volovich, Mathematical foundations of quantum information and computation and its applications to nano-and bio-systems: Springer Science \& Business Media, 2011.

[19] D. Marcuse, Principles of quantum electronics: Elsevier, 2012.

[20] R. Dorner and V. Vedral, "Correlations in Quantum Physics," arXiv preprint arXiv:1208.4961, 2012.

[21] F. Weinhold, "Metric geometry of equilibrium thermodynamics," The Journal of Chemical Physics, vol. 63, pp. 2479-2483, 1975.

[22] B. Chow and D. Knopf, The Ricci flow: an introduction vol. 110: American mathematical society Providence, 2004.

[23] R. S. Hamilton, "Three-manifolds with positive Ricci curvature," Journal of Differential Geometry, vol. 17, pp. 255-306, 1982.

[24] L. Landau, "EM. Lifshitz Statistical Physics," Course of Theoretical Physics, vol. 5, 1958.

[25] A. Schwenk and J. Polonyi, Renormalization group and effective field theory approaches to manybody systems vol. 852: Springer, 2012.

[26] C. Lin, "The Functional Determinant and the Partition Function in Geometric Flows," arXiv preprint arXiv:1311.5296, 2013. 
[27] D. Baumann and L. McAllister, Inflation and string theory: Cambridge University Press, 2015.

[28] M. Amiri, Tensor Properties of Joint Probability Densities on Riemannian Parametric Manifold. Preprints 2016, 2016100131 (doi: 10.20944/preprints201610. 0131.v1).

[29] S. Lovett, Differential Geometry of Manifolds, A K Peters/CRC Press, 2010 pp379

[30] Jacob D. Bekenstein, "Black holes and entropy." Physical Review D 7.8 (1973): 2333.

[31] Hawking, Stephen W. "Black hole explosions?." nature 248.5443 (1974): 30-31.

[32] C.Castro, (2008). The Euclidean gravitational action as black hole entropy, singularities, and spacetime voids. Journal of Mathematical Physics, 49(4), 042501.

[33] Tatsumi, Yuki, Tomoaki Kaneko, and Riichiro Saito. "Conservation law of angular momentum in helicity-dependent Raman and Rayleigh scattering." Physical Review B 97.19 (2018): 195444.

[34] Papoulis, Athanasios, and S. Unnikrishna Pillai. Probability, random variables, and stochastic processes. Tata McGraw-Hill Education, 2002.

[35] Troitskii, V. S. "Physical constants and evolution of the universe." Astrophysics and Space Science 139.2 (1987): 389-411.

[36] Cardenas, Ivan A., and Anton A. Lipovka. "Variation of the fine-structure constant caused by expansion of the Universe." Modern Physics Letters A 34.38 (2019): 1950315.

[37] Lipovka, Anton, and Iván A. Cárdenas. "Variation of the fine structure constant." (2016).

[38] Battaner, Eduardo, and Estrella Florido. "The rotation curve of spiral galaxies and its cosmological implications." arXiv preprint astro-ph/0010475 (2000). 